\title{
Absence of Phase Transitions in One-Dimensional Antiferromagnetic Models with Long-Range Interactions
}

\author{
Azer Kerimov ${ }^{1,2}$
}

Received August 4, 1992; final April 16, 1993

The absence of phase transitions in a one-dimensional model with long-range antiferromagnetic potential is established at low temperatures when the ground states have a rational density. A description of the set of all ground states and typical configurations is given.

KEY WORDS: Hamiltonian; ground state; Gibbs state; interacting and noninteracting contours; extreme Gibbs state.

\section{INTRODUCTION}

We consider a classical lattice model of statistical mechanics on a onedimensional lattice, the spin variable $\varphi(x)$ at each point $x$ taking the values 0 and 1 . The interaction is specified by means of the Hamiltonian

$$
\mathbf{H}(\varphi(x))=\sum_{x, y \in \mathbf{Z}^{1} ; x>y} U(x-y) \varphi(x) \varphi(y)-\mu \sum_{x \in \mathbf{Z}^{1}} \varphi(x)
$$

where $\mu$ is the external field. The following conditions are imposed on the potential $U(x)$ :

1. $U(x)>0$ at $x \in \mathbf{Z}^{1}, x>0$.

2. $\sum_{x \in \mathbf{Z}^{1}} U(x)<\infty$.

3. $U(x+y)+U(x-y)>2 U(x) ; x, y \in \mathbf{Z}^{1}, x>y$.

${ }^{1}$ Depmartment of Mathematics, Bilkent University, 06533 Bilkent, Ankara, Turkey.

${ }^{2}$ Permanent address: Institute of Mathematics and Mechanics, Academy of Sciences of Azerbaijan, Baku 370602, Azerbaijan. 
4. The function $U(x)$ can be extended to a twice continuously differentiable function such that $U(x) \sim A x^{-\gamma}, U^{\prime} \sim-A \gamma x^{-\gamma-1}$, and $U^{\prime \prime}(x) \sim A \gamma(\gamma+1) x^{-\gamma-2}$ at $x \rightarrow \infty$, where $\gamma>1$, and $A$ is a strong positive constant.

The first condition means that the model (1) is antiferromagnetic. The natural second condition is necessary for the existence of the thermodynamic limit. The third condition on the convexity of the interaction function $U(x)$ is essential for all further evaluations. The fourth condition determines the character of the potential's decrease at infinity.

The main purpose of the present paper is to investigate the structure of the set of all Gibbs states ${ }^{(1)}$ of the model (1).

The hypothesis on the uniqueness of the Gibbs states in the model (1) was stated by Sinai in 1983 (see ref. 2, Problem 1).

It is well known that the condition $\sum_{x \in \mathbf{Z}^{1}, x>0} x U(x)<\infty$ automatically implies the uniqueness of the Gibbs states. ${ }^{(3-5)}$ Therefore, we investigate the problem of the phase transitions in the model (1) for potentials $U(x) \sim A x^{-\gamma}$, where $\gamma=1+\alpha, 0<\alpha<1$. The ferromagnetic case [when the potential $U(x)$ is negative] was considered by Dyson. ${ }^{(6,7)} \mathrm{He}$ considered a model with the following potential [the external field is absent, spin variable $\varphi(x)$ takes the values $+1,-1]$ :

1. $U(x)<0$.

2. $\sum_{x \in \mathbf{Z}^{1}}|U(x)|<\infty$.

3. $U(x+1)>U(x)$.

4. $\sum_{x \in \mathbf{Z}^{1}, x>0} \ln \ln (x+4)^{-1} x^{3} U(x)^{-1}<\infty$.

Note that all potentials decreasing as $x^{-1-\alpha}, 0<\alpha<1$, certainly satisfy the above conditions.

Dyson established that in the ferromagnetic case one can find $\beta_{1}$ such that if $\beta>\beta_{1}$ then there exist at least two extremal Gibbs states $P^{+}$and $P^{-}$ corresponding to the ground states $\varphi(x)=+1$ and $\varphi(x)=-1$. This very profound result is connected with the following fact. Let us consider the boundary conditions $\bar{\varphi}(x)=1$, the segment $[-n, n]$, and the configuration $\varphi_{-1}(x)$ such that $\varphi_{-1}(x)=-1$ if $x \in[-n, n]$, and $\varphi_{-1}(x)=1$ if $x \in \mathbf{Z}^{1}-$ $[-n, n]$. Then the difference between the energies of the configurations $\varphi_{-1}(x)$ and $\bar{\varphi}(x)$ is of order $n^{1-x}$. In other words, in the one-dimensional case there arises an analog of the notion of the surface tension and this fact leads to the existence of two extremal Gibbs states, as could be anticipated.

In the antiferromagnetic case we are faced with a quite different situation. It will be shown that at arbitrary fixed boundary conditions $\bar{\varphi}(x), x \in \mathbf{Z}^{1}-[-n, n]$, a configuration $\varphi(x), x \in[-n, n]$, with maximal 
weight (or with minimal energy) almost does not differ from the special ground state with the exception of some bounded zone and is stable in the sense of Peierls (see Lemma 5). This fact has a decisive significance in establishing all further results.

A series of papers has been devoted to the investigation of the ground states of the model $(1) .^{(8-10,2,11-14)}$

Let us now briefly introduce necessary definitions and facts. Let $\Phi^{\text {per }}$ denote the set of all periodic configurations. For every $\varphi \in \Phi^{\text {per }}$ we define $q=\sum_{y=x+1}^{x+p} \varphi(x) / p$, where $p$ is the period of $\varphi$. It is obvious that $q$ does not depend on $x$. Therefore, the density of each periodic configuration is $\kappa=q / p$. It is more convenient to work with the reciprocal of the density, $\eta(\varphi(x))=p / q$, which represents the average distance between neighboring points at which $\varphi(x)=1$. For every configuration $\varphi \in \Phi^{\text {per }}$ we define the mean energy $h(\varphi)$ as follows:

$$
h(\varphi(x))=\frac{1}{p} \sum_{y=x+1}^{x+p} \varphi(x) \sum_{z>0} U(z) \varphi(y+z)
$$

It is readily seen that this expression is independent of $x$.

The special definition of the ground state was formulated in ref. 2. This definition does not coincide with the generally accepted one ${ }^{(1)}$ and is useful for describing the phase diagram of the model (1) at zero temperature.

Let $p / q$ be a fixed positive rational number.

Definition 1. ${ }^{(2)}$ A configuration $\varphi_{0}(x) \in \Phi^{\text {per }}$ with $\eta\left(\varphi_{0}(x)=p / q\right)$ is called a special ground state if

$$
h(\varphi(x))=\inf _{\varphi \in \Phi \operatorname{per}, \eta(\varphi)=p / q} h(\varphi)
$$

The following proposition readily follows from the convexity of the potential $U(x)$.

Hubbard's Criterion. ${ }^{(2,8)}$ Let $\varphi \in \Phi^{\text {per }}$ and let $r_{i}(x ; \varphi)$ denote the distance between $x \in \mathbf{Z}^{1}$ and the $i$ th particle on the right. If for each $x$ and $i$

$$
[i \eta] \leqslant r_{i}(x ; \varphi) \leqslant[i \eta]+1
$$

(the square brackets denote the integral part of the enclosed number) then $\varphi$ is a special ground state.

The existence of a configuration satisfying Hubbard's criterion (the special ground state) is proved in ref. 2. A remarkable short formula for the special ground states was given by S. Aubry (see ref. 13). Here we give the construction of the special ground state for each fixed rational 
value of the density $\kappa \cdot{ }^{(2)}$ Special ground states for irrational densities are investigated in refs. 13 and 14.

Every rational number $p / q$ can be uniquely decomposed into a finite continued fraction. We write it as $p / q=\left[n_{0}, n_{1}, \ldots, n_{s}\right]$, which means

$$
n_{0}+\frac{1}{n_{1}+\frac{1}{n_{2}+\cdots+\frac{1}{n_{s}}}}
$$

The ground state for a configuration with $\kappa=\left[n_{0}, n_{1}, \ldots, n_{s}\right]$ will be constructed by induction.

1. $\kappa=n_{0} \geqslant 1, n_{1}$ is an integer. It is then obvious that a periodic configuration with equally distant $x$ at which $\varphi(x)=1$ satisfies Hubbard's criterion, i.e., is a special ground state. In this case $r_{i}(x ; \varphi)=i n_{0}, i>0$.

2. $\kappa=n_{0}+1 / n_{1}$, where $n_{0}$ and $n_{1}$ are integers, $n_{0} \geqslant 1, n_{1}>1$. Then the $\left(n_{0} n_{1}+1\right)$-periodic configuration

$$
\underbrace{0 \ldots 01}_{n_{0}+1} \underbrace{0.01}_{\underbrace{0.001}_{n_{1}-1 \text { times }} \ldots \underbrace{0 . \ldots 01}_{n_{0}}}
$$

also satisfies Hubbard's criterion and is a special ground state.

3. $\kappa=\left[n_{0}, n_{1}, \ldots, n_{s}\right]$, where $n_{0}, n_{1}, \ldots, n_{s}$ are integers, $n_{0}, n_{1}, \ldots, n_{s} \geqslant 1$. For $s=0$ and $s=1$ the required configurations are already constructed. Suppose we have already constructed a ground state with $s=m$ and $\kappa=\left[n_{0}, n_{1}, \ldots, n_{m}\right]$. Then the following configuration with $s=m+1$ and $\kappa=\left[n_{0}, n_{1}, \ldots, n_{m+1}\right]$ is constructed:

$$
\varphi\left(n_{0}, \ldots, n_{m+1}\right)=\varphi\left(n_{0}, \ldots, n_{m-1}\right) \underbrace{\varphi\left(n_{0}, \ldots, n_{m}\right) \cdots \varphi\left(n_{0}, \ldots, n_{m}\right)}_{n_{m+1} \text { times }}
$$

Here, $\varphi\left(n_{0}, \ldots, n_{j}\right), j=m-1, m, m+1$, are the blocks from which the ground states for $\kappa=\left[n_{0}, \ldots, n_{j}\right]$ are obtained by periodic continuations.

It can be verified ${ }^{(2)}$ that the constructed configuration satisfies Hubbard's criterion and therefore is a special ground state for $\kappa=\left[n_{0}, n_{1}, \ldots, n_{m}, n_{m+1}\right]$.

Hubbard's criterion allows us to extract an explicit expression for the mean energy of a special ground state ${ }^{(2)}$ :

$$
h_{\kappa}=\kappa \sum_{i=1}^{\infty} U\left(m_{i}\right) \pi_{i}+U\left(m_{i}+1\right)\left(1-\pi_{i}\right)
$$

where $m_{i}=[i \eta], \pi_{i}=1+m_{i}-i \eta$. 
This formula shows that the function of mean energy is continuous on the set of all rationals and can be extended to a continuous function defined on the whole segment $[0,+\infty)$.

Theorem 1..$^{(2,10)}$ 1. The function $h_{\kappa}$ is convex.

2. In each rational point the function $h_{\kappa}$ has a left-hand derivative $\mu_{\kappa}^{-}$and a right-hand derivative $\mu_{\kappa}^{+}$, with $\mu_{\kappa}^{+}>\mu_{\kappa}^{-}$.

3. The Lebesgue measure of the complement of the set $U_{\kappa}\left(\mu_{\kappa}^{-}, \mu_{\kappa}^{+}\right)$ in the real line $\mathbf{R}$ is zero.

Theorem 2. ${ }^{(11)}$ Suppose that the value of the external field $\mu$ of the model (1) belongs to the interval $\left(\mu_{\kappa}^{-}, \mu_{\kappa}^{+}\right)$for some number $\kappa=q / p$. Then the special ground state of the model (1) is unique to within a translation.

In this paper we establish the validity of Sinai's hypothesis at low temperatures almost (with respect to the Lebesgue measure) for each value of the external field. The main result of the present paper (and the solution of Problem 1 of ref. 2 almost for each value of the external field) is the following:

Theorem 3. Suppose that the value of the external field $\mu$ of the model (1) belongs to the interval $\left(\mu_{\kappa}^{-}, \mu_{\kappa}^{+}\right)$for some number $\kappa=q / p$.

Then the model (1) has a unique Gibbs state at all sufficiently small values of the temperature $\left[\beta^{-1}<\operatorname{const}(\mu, U(x))\right]$.

Suppose that the value of the external field $\mu$ of the model (1) belongs to the interval $\left(\mu_{\kappa}^{-}, \mu_{\kappa}^{+}\right)$for some number $\kappa=q / p$.

Let us consider an arbitrary configuration $\varphi(x)$. We say that $\varphi(V)$, $V \in \mathbf{Z}^{1}$, is a preregular phase if there exists a special ground state $\varphi_{\kappa}$ such that a restriction of this configuration on $V$ coincides with $\varphi(V)$. We say that $\varphi\left(V^{\prime}\right), V^{\prime} \in \mathbf{Z}^{1}$, is a regular phase if there exists a preregular phase $\varphi(V), V \in \mathbf{Z}^{1}$, such that both $V^{\prime}+d_{0} p$ and $V^{\prime}-d_{0} p$ belong to $V$.

Let us consider a set $A=\bigcup_{i} V_{i}$, where $\varphi\left(V_{i}\right)$ is a regular phase and supp $P B$ is a complement of $A$ in $Z^{1}$. The connected components of supp $P B$ defined in such a way are called supports of precontours and are denoted by supp $P K$.

The value of the constant $d_{0}$ will be defined later.

Definition. 2. The pair $P K=\left(\operatorname{supp} P K, \varphi^{\prime}(\operatorname{supp} P K)\right)$ is called a precontour. The set of all precontours is called a preboundary $P B$ of the configuration $\varphi^{\prime}(x)$. Two precontours $P K_{1}$ and $P K_{2}$ are said to be connected if $\operatorname{dist}\left(\operatorname{supp} P K_{1}, \operatorname{supp} P K_{2}\right)<N_{b}$. The set of precontours 
$\left(P K_{i} ; i \in I\right)$ is called connected if for any two precontours $P K_{p}$ and $P K_{q}$, $p, q \in I$ there exists a collection

$$
\begin{gathered}
\left(P K_{(j, 1)}=P K_{p}, \ldots, P K_{(j, i)}, \ldots, P K_{(j, n-1)}, P K_{(j, n)}=P K_{q}\right) \\
(j, i) \in I, \quad i=1, \ldots, n
\end{gathered}
$$

such that any two precontours $P K_{(j, i)}$ and $P K_{(j, i+1)}, i=1, \ldots, n-1$, are connected. Let $\bigcup_{i=1}^{n} P K_{i}$ be some maximal connected component of the preboundary $P B$. Suppose that $\operatorname{supp} P K_{i}=\left[a_{i}, b_{i}\right]$ and $b_{i}<a_{i+1}$, $i=1, \ldots, n-1$.

The pair $K=\left(\operatorname{supp} K, \varphi^{\prime}(\operatorname{supp} P K)\right)$, where $\operatorname{supp} K=\left[a_{1}, b_{n}\right]$ is called a contour. The set of all contours is called a boundary $\mathbf{B}$ of the configuration $\varphi^{\prime}(x)$.

The value of the constant $N_{b}$ will be defined later.

Note that

$$
\begin{aligned}
\operatorname{supp} K & =\left(\bigcup_{i=1}^{n} \operatorname{supp} P K_{i}\right) \cup\left(\left[a_{1}, b_{n}\right]-\left(\bigcup_{i=1}^{n} \operatorname{supp} P K_{i}\right)\right) \\
& =\operatorname{supp}^{1} K \cup \operatorname{supp}^{2} K
\end{aligned}
$$

The sets $\operatorname{supp}^{1} K$ and $\operatorname{supp}^{2} K$ will be respectively called the essential and regular parts of the support supp $K$.

Let the boundary conditions $\bar{\varphi}(x)=[\varphi(x), x \in(-\infty,-V-1] \cup$ $[V+1, \infty)]$ be fixed. A set of all configurations $\varphi(x), x \in[-V, V]$, we denote by $\Phi(V)$.

It is obvious that for each contour $K$ such that supp $K \in\left[-V+\left(d_{0}+1\right) p\right.$, $\left.V-\left(d_{0}+1\right) p\right]$ there exists a configuration $\psi_{K}([-V, V])$ such that the boundary of the configuration $\psi_{K}([-V, V])$ includes the contour $K$ only:

$$
P B\left(\psi_{K}([-V, V])\right)=\dot{K}
$$

This means that the configuration $\psi_{K}([-V, V])$ contains one contour $K$ and two regular phases $\psi^{1}(x)$ and $\psi^{2}(x)$. It is obvious that there exist unique special ground states $\varphi_{\kappa}^{1}(x)$ and $\varphi_{\kappa}^{2}(x)$ such that restrictions of the configurations $\varphi_{\kappa}^{1}(x)$ and $\varphi_{\kappa}^{2}(x)$ on the supports of the regular phases $\psi^{1}(x)$ and $\psi^{2}(x)$ respectively coincide with the $\psi^{1}(x)$ and $\psi^{2}(x)$.

Definition 3. A contour $K$ is called an interface contour if $\varphi_{\kappa}^{1}(x) \neq \varphi_{\kappa}^{2}(x)$. $\varphi_{\kappa}^{2}$.

Note that $\varphi_{\kappa}^{1}(x)$ can be obtained by some shifting of the configuration An interface contour will be denoted as $I K$. 
Let $K$ be the usual contour $K$ and $\psi_{K}(x)=\psi([-V, V])$ if $x \in[-V, V]$, and $\bar{\varphi}(x)$ if $x \in(-\infty,-V-1] \cup[V+1, \infty)$; let $I K$ be an interface contour and $\psi_{I K}(x)=\psi([-V, V])$ if $x \in[-V, V]$, and $\bar{\varphi}(x)$ if $x \in(-\infty,-V-1] \cup[V+1, \infty) ; \bar{\varphi}_{\kappa}(x)=\varphi_{n_{0}}(x)$ if $x \in[-V, V]$, and $\bar{\varphi}(x)$ if $x \in(-\infty,-V-1] \cup[V+1, \infty)$; and $\bar{\varphi}_{\kappa}^{1}(x)=\varphi_{\kappa}^{1}(x)$ if $x \in[-V, V]$ and $\bar{\varphi}(x)$ if $x \in(-\infty,-V-1] \cup[V+1, \infty)$.

The weights of the usual contour $K$ and interface contour $I K$ will be calculated by the following formulas:

$$
\begin{gathered}
\gamma(K)=H\left(\psi_{K}(x)\right)-H\left(\bar{\varphi}_{\kappa}(x)\right) \\
\gamma(I K)=H\left(\psi_{I K}(x)\right)-H\left(\bar{\varphi}_{\kappa}^{1}(x)\right)
\end{gathered}
$$

For establishing the uniqueness of the Gibbs states at low temperatures in the model (1) we use the following strategy. First, we prove that at low temperatures the typical configurations of the Gibbs state $\mathbf{P}^{1}$ corresponding to the boundary conditions $\varphi^{1}(x)$ are small perturbations of the special ground states separated by a possibly finite number of interfaces. Second, we consider an arbitrary configuration $\varphi^{\prime}(I)$, with $I$ being an arbitrary segment, and a sufficiently large volume $V$, and establish that the dependence of the expression $\mathbf{P}^{1}\left(\varphi^{\prime}(I)\right): \mathbf{P}^{2}\left(\varphi^{\prime}(I)\right)$ on the boundary conditions $\varphi^{1}(x)$ and $\varphi^{2}(x)$ can be estimated through the sum of statistical weights of unlikely clusters connecting the segment $I$ with the boundary. Finally, by using the Peierls estimation, we prove that the sum of weights of these clusters is a finite number, not depending on $\varphi^{\prime}(I), V, \varphi^{1}(x)$, and $\varphi^{2}(x)$. Thus, two arbitrary extreme Gibbs states are relatively continuous and hence coincide.

The contents of this paper are as follows. In Sections 2-4 we assume that the density of the special ground state is $1 / n_{0}$. In Section 2 the set of all ground states of the model (1) is studied. In Section 3, Gibbs states are investigated and the Peierls estimation is proved. In Section 4 the uniqueness of Gibbs states is established. In Section 5 all obtained results are generalized for all rational values of the density.

\section{GROUND STATES. THE DENSITY $\mathrm{k}$ IS $1 / n_{0}$}

In this section we continue to study the set of all ground states and give a description of this set for special values of the external field (when the density of the special ground state is $1 / n_{0}$ ). The general case will be considered in Section 4.

Now we start to estimate the weight of a contour which contains only one precontour. 
Lemma 1. Suppose that the value of the external field $\mu$ of the model (1) belongs to the interval $\left(\mu_{\kappa}^{-}, \mu_{\kappa}^{+}\right)$for some number $\kappa=q / p=1 / n_{0}$. Let $\varphi^{\prime}(x)$ be an arbitrary finite perturbation of the special ground state $\varphi_{n_{0}}(x)$ such that the boundary $P B$ of the configuration $\varphi^{\prime}(x)$ includes a unique precontour $P K$. Then there exists a positive constant $t$ depending only on the Hamiltonian (1) such that

$$
H\left(\varphi^{\prime}(x)\right)-H\left(\varphi_{n_{0}}(x)\right) \geqslant t|\operatorname{supp} P B|
$$

where $\mid$ supp $P B \mid$ is the total area of the support of the boundary.

Proof. 1. Suppose that $\sum_{x \in \operatorname{supp} K}\left(\varphi^{\prime}(x)-\varphi_{n_{0}}(x)\right)=0$. This means that a perturbation $\varphi(x)$ is obtained by shifting of some particles only.

If the length of the support of a contour is $|\operatorname{supp} K|=L$, then, according to the definition of a contour, the contour $K$ contains at most $d_{0}\left(L+n_{0}\right):\left(d_{0}+1\right) n_{0}$ number of blocks

$$
\varphi_{0}=\underbrace{0 \ldots 01}_{n_{0}}
$$

Then the convexity of $U(x)$ directly leads to the required estimation (6) with $^{(2)}$

$$
\begin{aligned}
& t=t_{0}=\left(U\left(n_{0}-1\right)+U\left(n_{0}+1\right)-2 U\left(n_{0}\right)\right) \theta \\
& \theta=\left(L-d_{0} n_{0}\right): 2 L\left(d_{0}+1\right) n_{0}
\end{aligned}
$$

2. Suppose that $\sum_{x \in \operatorname{supp} K}\left(\varphi^{\prime}(x)-\varphi(x)\right) \neq 0$. Let the density of the configuration $\varphi^{\prime}(x), x \in \operatorname{supp} K$, be $\kappa$ and the reciprocal of the density be $\eta$. Now we divide the proof of this section into six cases.

Case $2 a . \quad n_{0}+1 \leqslant \eta$.

Case 2b. $n_{0}+1-\varepsilon<\eta<n_{0}+1$.

Case 2c. $n_{0}<\eta \leqslant n_{0}+1-\varepsilon$.

Case $2 d . \quad \eta \leqslant n_{0}-1$.

Case 2e. $n_{0}-1<\eta<n_{0}-1+\delta$.

Case 2f. $n_{0}-1+\delta \leqslant \eta<n_{0}$.

The constants $\varepsilon$ and $\delta$ will be defined later.

Case $2 a . \quad n_{0}+1 \leqslant \eta$. The density of the configuration $\varphi^{\prime}(x)$ is $\kappa=\eta^{-1}$. Note that 


$$
\begin{aligned}
& H\left(\varphi^{\prime}(x)\right)-H\left(\varphi_{n_{0}}(x)\right) \\
& =\sum_{x, y \in \mathbf{Z}^{1}, x>y ; x, y \in \operatorname{supp} K} U(x-y)\left(\varphi^{\prime}(x) \varphi^{\prime}(y)-\varphi_{n_{0}}(x) \varphi_{n_{0}}(y)\right) \\
& +\sum_{x, y \in \mathbf{Z}^{1}, x>y ; x \in \operatorname{supp} K, y \notin \operatorname{supp} K \text { or } x \notin \operatorname{supp} K, y \in \operatorname{supp} K} U(x-y) \\
& \times\left(\varphi^{\prime}(x) \varphi^{\prime}(y)-\varphi_{n_{0}}(x) \varphi_{n_{0}}(y)\right) \\
& -\mu \sum_{x \in \mathbf{Z}^{\mathrm{f}}, x \in \operatorname{supp} K}\left(\varphi^{\prime}(x)-\varphi_{n_{0}}(x)\right) \\
& =\mathbf{A}_{1}+\mathbf{B}_{1}+\mathbf{C}_{1} \\
& \mathbf{A}_{1}+\mathbf{B}_{1} \geqslant \mathbf{A}_{1} \\
& +\sum_{x, y \in \mathbf{Z}^{1}, x>y ; x \in \operatorname{supp} K, y \notin \operatorname{supp} K \text { or } x \notin \operatorname{supp} K, y \in \operatorname{supp} K} U(x-y) \\
& \times\left(\varphi^{\prime \prime}(x) \varphi^{\prime \prime}(y)-\varphi_{n_{0}}(x) \varphi_{n_{0}}(y)\right) \\
& \geqslant \sum_{x, y \in \mathbf{Z}^{1}, x>y ; x, y \in \operatorname{supp} K} U(x-y)\left(\varphi_{\eta}(x) \varphi_{\eta}(y)-\varphi_{n_{0}}(x) \varphi_{n_{0}}(y)\right) \\
& +\sum_{x, y \in \mathbf{Z}^{1}, x>y ; x \in \operatorname{supp} K, y \notin \operatorname{supp} K \text { or } x \notin \operatorname{supp} K, y \in \operatorname{supp} K} U(x-y) \\
& \times\left(\varphi_{\eta}(x) \varphi_{\eta}(y)-\varphi_{n_{0}}(x) \varphi_{n_{0}}(y)\right) \\
& =\mathbf{A}_{1}^{\prime}+\mathbf{B}_{1}^{\prime}
\end{aligned}
$$

Here the function $\varphi^{\prime \prime}(x)=\varphi_{\eta}(x)$ if $x \notin \operatorname{supp} K$ and $\varphi^{\prime}(x)$ if $x \in \operatorname{supp} K$; and the function $\varphi_{\eta}(x)$ is a special ground state with the density $\kappa=\eta^{-1}$.

The first inequality is valid due to the following fact:

Let $\varphi_{n_{0}}(x), x \in[N, \infty]$, and $\varphi_{\eta}, x \in[N, \infty]$, be restrictions of special ground states with the densities $n_{0}^{-1}$ and $\kappa$, respectively, on an arbitrary segment $[N, \infty], N>0\left(\eta>n_{0}\right)$. Then

$$
\sum_{x \in \mathbf{Z}^{1} ; x \geqslant N} U(x)\left(\varphi_{n_{0}}(x)-\varphi_{\eta}(x)\right) \geqslant 0
$$

The last inequality shows that the "influence" of the boundary conditions $\varphi_{n_{0}}(x)$ is stronger than the "influence" of the boundary conditions $\varphi_{\eta}(x)$.

The validity of the second inequality is a direct implication of the convexity of $U(x){ }^{(2)}$

Note that

$$
\begin{aligned}
\mathbf{C}_{1} & =\mu \sum_{x \in \mathbf{Z}^{\prime}, x \in \operatorname{supp} K}\left(\varphi^{\prime}(x)-\varphi_{n_{0}}(x)\right) \\
& =\mu \sum_{x \in \mathbf{Z}^{1}, x \in \operatorname{supp} K}\left(\varphi_{\eta}(x)-\varphi_{n_{0}}(x)\right)=\mathbf{C}_{1}^{\prime}
\end{aligned}
$$


Finally

$\mathbf{A}_{1}+\mathbf{B}_{1}+\mathbf{C}_{1} \geqslant \mathbf{A}_{1}^{\prime}+\mathbf{B}_{1}^{\prime}+\mathbf{C}_{1}^{\prime}$

$$
\begin{gathered}
=\sum_{x, y \in \mathbf{Z}^{\mathrm{l}}, x>y ; x \text { or } y \in \operatorname{supp} K} U(x-y)\left(\varphi_{\eta}(x) \varphi_{\eta}(y)-\varphi_{n_{0}}(x) \varphi_{n_{0}}(y)\right) \\
-\mu \sum_{x \in \mathbf{Z}^{1}, x \in \operatorname{supp} K}\left(\varphi_{\eta}(x)-\varphi_{n_{0}}(x)\right)=\mathbf{Q}
\end{gathered}
$$

By using the definition of the mean energy we get $\left(\kappa=\eta^{-1}\right.$, $\left.\kappa_{0}=n_{0}^{-1}=q / p\right)$

$$
\begin{aligned}
\mathbf{Q} & =\left(h_{\kappa}-h_{\kappa_{0}}\right)|\operatorname{supp} K|-\mu\left(\kappa-\kappa_{0}\right)|\operatorname{supp} K| \\
& =\left(h_{\kappa}-h_{\kappa_{0}}-\mu\left(\kappa-\kappa_{0}\right)\right)|\operatorname{supp} K|
\end{aligned}
$$

Let us consider the expression $h_{\kappa}-h_{\kappa_{0}}-\mu\left(\kappa-\kappa_{0}\right)$. According to Theorem 2, the function $h_{\kappa}$ is convex and has a left-hand derivative $\mu_{\kappa}^{-}$at the point $\kappa$. Therefore,

$$
h_{\kappa}-h_{\kappa_{0}} \geqslant \mu_{\kappa}^{-}\left(\kappa-\kappa_{0}\right) \geqslant \mu\left(\kappa-\kappa_{0}\right)
$$

The last inequality is valid due to the assumption $\mu \in\left(\mu_{\kappa}^{-}, \mu_{\kappa}^{+}\right)$(note that $\kappa-\kappa_{0}$ is negative and less than one). Finally, the formulas (8)-(10) lead to the required estimation with

$$
t=t_{\eta}=\mu_{\kappa}\left(\kappa_{0}-\kappa\right)-\left(h_{\kappa_{0}}-h_{\kappa}\right)
$$

Therefore, taking into account the condition $n_{0}+1 \leqslant \eta$, we see that in Case $2 \mathrm{a}$ the required estimation (6) holds at

$$
t=t_{1}=\min _{n_{0}+1 \leqslant \eta} t_{\eta}=\mu_{\kappa}\left(\kappa_{0}-\left(\kappa_{0}^{-1}+1\right)^{-1}\right)-\left(h_{\kappa_{0}}-h_{\left(\kappa_{0}^{-1}+1\right)^{-1}}\right)
$$

The proof of Case $2 \mathrm{a}$ is completed.

Case 2b. $n_{0}+1-\varepsilon<\eta<n_{0}+1$. The density of the configuration $\varphi^{\prime}(x)$ is $\kappa=\eta^{-1}$. If $n_{0}+1 \leqslant \eta$ (Case $2 \mathrm{a}$ ), then [see (8)-(10)]

$$
\begin{aligned}
H\left(\varphi^{\prime}(x)\right)-H\left(\varphi_{n_{0}}(x)\right) & \geqslant \mathbf{Q} \\
& =\left(h_{\kappa}-h_{\kappa_{0}}-\mu\left(\kappa-\kappa_{0}\right)\right)|\operatorname{supp} K| \geqslant t_{2}|\operatorname{supp} K|
\end{aligned}
$$

For a function of mean energy $h_{\kappa}$ we have an explicit expression which shows that $h_{\kappa}$ is continuous. Therefore the function $h_{\kappa}-h_{\kappa_{0}}-\mu\left(\kappa-\kappa_{0}\right)$ is 
continuous particularly at the point $\left(\kappa_{0}^{-1}+1\right)^{-1}$. Then there exists $\varepsilon_{1}>0$ such that for all $\kappa:\left|\kappa-\left(\kappa_{0}^{-1}+1\right)^{-1}\right|<\varepsilon_{1}$

$$
\left(h_{\kappa}-h_{\kappa_{0}}-\mu\left(\kappa-\kappa_{0}\right)\right)|\operatorname{supp} K| \geqslant t_{2} / 2|\operatorname{supp} K|
$$

Finally, $\varepsilon=\varepsilon_{1}, t_{2}=t_{1} / 2$.

The proof of Case $2 b$ is completed.

Case 2c. $n_{0}<\eta \leqslant n_{0}+1-\varepsilon$. The density of the configuration $\varphi^{\prime}(x)$ is $\kappa=\eta^{-1}$. Similarly to Case $2 \mathrm{a}$, we get [see (8)-(10)]

$$
\begin{gathered}
H\left(\varphi^{\prime}(x)\right)-H\left(\varphi_{n_{0}}(x)\right)=\mathbf{A}_{1}+\mathbf{B}_{1}+\mathbf{C}_{1} \\
\mathbf{A}_{1}^{\prime}+\mathbf{B}_{1}^{\prime}+\mathbf{C}_{1}^{\prime}+\mathbf{B}_{1}+\mathbf{C}_{1}=\left(\mathbf{A}_{1}+\mathbf{B}_{1}-\mathbf{A}_{1}^{\prime}-\mathbf{B}_{1}^{\prime}\right)+\mathbf{A}_{1}^{\prime}+\mathbf{B}_{1}^{\prime}+\mathbf{C}_{1}^{\prime} \\
=\sum_{x, y \in \mathbf{Z}^{1}, x>y ; x \text { or } y \in \operatorname{supp} K} U(x-y)\left(\varphi_{\eta}(x) \varphi_{\eta}(y)-\varphi_{n_{0}}(x) \varphi_{n_{0}}(y)\right) \\
\quad-\sum_{x \in \mathbf{Z}^{1}, x \in \operatorname{supp} K}\left(\varphi_{\eta}(x)-\varphi_{n_{0}}(x)\right)=\mathbf{Q}
\end{gathered}
$$

By using the definition of the mean energy we get $\left(\kappa=\eta^{-1}, \kappa_{0}=\right.$ $\left.n_{0}^{-1}=q / p\right)$

$$
\begin{aligned}
\mathbf{Q} & =\left(h_{\kappa}-h_{\kappa_{0}}\right)|\operatorname{supp} K|-\mu\left(\kappa-\kappa_{0}\right)|\operatorname{supp} K| \\
& =\left(h_{\kappa}-h_{\kappa_{0}}-\mu\left(\kappa-\kappa_{0}\right)\right)|\operatorname{supp} K|
\end{aligned}
$$

Let us consider the expression $h_{\kappa}-h_{\kappa_{0}}-\mu\left(\kappa-\kappa_{0}\right)$. According to Theorem 2, the function $h_{\kappa}$ is convex and has a right-hand derivative $\mu_{\kappa}^{+}$ at the point $\kappa$. Therefore

$$
h_{\kappa}-h_{\kappa_{0}} \geqslant \mu_{\kappa}^{+}\left(\kappa-\kappa_{0}\right) \geqslant \mu\left(\kappa-\kappa_{0}\right)
$$

The last inequality is valid due to the assumption $\mu \in\left(\mu_{\kappa}^{-}, \mu_{\kappa}^{+}\right)$.

Note that in Case $2 c$ the inequality (13) does not give us the required estimation (6) because

$$
\min _{n_{0}<\eta \leqslant n_{0}+1-\varepsilon}\left(h_{\kappa}-h_{\kappa_{0}}-\mu\left(\kappa-\kappa_{0}\right)\right)=0
$$

Let us consider the expression $\left(\mathbf{A}_{1}+\mathbf{B}_{1}-\mathbf{A}_{1}^{\prime}-\mathbf{B}_{1}^{\prime}\right)$.

If the length of the support of a precontour is $\mid$ supp $P K \mid=L$, then according to the definition of a precontour, the precontour $P K$ contains at most $d_{0}\left(L+n_{0}\right):\left(d_{0}+1\right) n_{0}$ number of blocks

$$
\varphi_{0}=\underbrace{0 \ldots 01}_{n_{0}}
$$


Then the convexity of $U(x)$ directly leads to the estimation

$$
\mathbf{A}_{2}+\mathbf{B}_{2}-\mathbf{A}_{2}^{\prime}-\mathbf{B}_{2}^{\prime} \geqslant t_{\eta} \theta|\operatorname{supp} K|
$$

where

$$
\begin{aligned}
t_{\eta} & =1 / 2\left(1+n_{0}-\eta\right)\left(U\left(n_{0}-1\right)+U\left(n_{0}+1\right)-2 U\left(n_{0}\right)\right) \\
\theta & =\left(L-d_{0} n_{0}\right): 2 L\left(d_{0}+1\right) n_{0}
\end{aligned}
$$

Finally, the formulas (15)-(20) lead to the inequality (6) with $t=t_{\eta}$.

Therefore, taking into account the condition $n_{0}<\eta \leqslant n_{0}+1-\varepsilon$, we see that in Case $2 c$ the required estimation (6) holds at

$$
\begin{aligned}
t & =t_{3}=\min _{\eta \in\left(n_{0}, n_{0}+1-\varepsilon\right]} 1 / 2\left(1+n_{0}-\eta\right)\left(U\left(n_{0}-1\right)+U\left(n_{0}+1\right)-2 U\left(n_{0}\right)\right) \theta \\
& =1 / 2\left(U\left(n_{0}-1\right)-2 U\left(n_{0}\right)+U\left(n_{0}+1\right)\right) c \theta
\end{aligned}
$$

Case $2 d$. $\eta \leqslant n_{0}-1$. The density of the configuration $\varphi^{\prime}(x)$ is $\kappa=\eta^{-1}$. Similarly to Case 2a, we get [see formula (8)]

$$
H\left(\varphi^{\prime}(x)\right)-H\left(\varphi_{n_{0}}(x)\right)=\mathbf{A}_{1}+\mathbf{B}_{1}+\mathbf{C}_{1}
$$

$\mathbf{A}_{1}+\mathbf{B}_{1} \geqslant \mathbf{A}_{1}$

$$
\begin{aligned}
& +\sum_{\begin{array}{c}
x, y \in \mathbf{Z}^{1}, x>y ; x \in \operatorname{supp} K, y \notin \operatorname{supp} K \text { or } x \notin \operatorname{supp} K, y \in \operatorname{supp} K \\
\end{array}} U(x-y) \\
& \times\left(\varphi^{\prime \prime}(x) \varphi^{\prime \prime}(y)-\varphi_{n_{0}}(x) \varphi_{n_{0}}(y)\right) \\
& \sum_{x, y \in \mathbf{Z}^{1}, x>y ; x, y \in \operatorname{supp} K} U(x-y) \\
& \times\left(\varphi_{\eta}(x) \varphi_{\eta}(y)-\varphi_{n_{0}}(x) \varphi_{n_{0}}(y)\right) \\
& +\sum_{x, y \in \mathbf{Z}^{1}, x>y ; x \in \operatorname{supp} K, y \notin \operatorname{supp} K \text { or } x \notin \operatorname{supp} K, y \in \operatorname{supp} K} U(x-y) \\
& \times\left(\varphi^{\prime \prime \prime}(x) \varphi^{\prime \prime \prime}(y)-\varphi_{n_{0}}(x) \varphi_{n_{0}}(y)\right) \\
& =\mathbf{A}_{1}^{\prime}+\mathbf{B}_{1}^{\prime}
\end{aligned}
$$

Here the function $\varphi^{\prime \prime}(x)=0$ if $x \notin \operatorname{supp} K$, and $\varphi^{\prime}(x)$ if $x \in \operatorname{supp} K$ and the function $\varphi^{\prime \prime \prime}(x)=0$ if $x \notin \operatorname{supp} K$, and $\varphi_{\eta}(x)$ if $x \in \operatorname{supp} K$.

The obvious first inequality shows that the "influence" of the boundary conditions $\varphi_{n_{0}}(x)$ is stronger than the "influence" of the empty boundary conditions.

The validity of the second inequality is a direct implication of the convexity of $U(x) .^{(2)}$ 
Note that

$$
\begin{aligned}
\mathbf{C}_{1} & =\mu \sum_{x \in \mathbf{Z}^{1}, x \in \operatorname{supp} K}\left(\varphi^{\prime}(x)-\varphi_{n_{0}}(x)\right) \\
& =\mu \sum_{x \in \mathbf{Z}^{1}, x \in \operatorname{supp} K}\left(\varphi^{\prime \prime \prime}(x)-\varphi_{n_{0}}(x)\right)=\mathbf{C}_{1}^{\prime}
\end{aligned}
$$

Finally

$$
\begin{aligned}
\mathbf{A}_{1}+\mathbf{B}_{1}+\mathbf{C}_{1} \geqslant & \mathbf{A}_{1}^{\prime}+\mathbf{B}_{1}^{\prime}+\mathbf{C}_{1}^{\prime} \\
= & \sum_{\substack{x, y \in \mathbf{Z}^{1}, x>y ; x \text { or } y \in \operatorname{supp} K\\
}} U\left(\varphi^{\prime \prime \prime}(x) \varphi^{\prime \prime \prime}(y)-\varphi_{n_{0}}(x) \varphi_{n_{0}}(y)\right) \\
& -\mu \sum_{x \in \mathbf{Z}^{1}, x \in \operatorname{supp} K}\left(\varphi^{\prime \prime \prime}(x)-\varphi_{n_{0}}(x)\right)=\mathbf{Q}
\end{aligned}
$$

Now, as in Case $2 \mathrm{a}$, we have to investigate the expression $\mathbf{Q}$.

But in this case the situation is slightly more difficult (not symmetric with Case $2 \mathrm{a}$ ). The fact is that in Case $2 \mathrm{~d}$, instead of the desired inequality (6) with the function $\varphi_{\eta}(x)$ we have the inequality (6) with the function $\varphi^{\prime \prime \prime}(x)$. Below (Lemma 2) we shall prove that the difference between these two functions is not so significant.

We introduce the following function:

$$
h_{\kappa}^{L}=\mu \sum_{x, y \in \mathbf{Z}^{1}, x>y ; x, y \in[-L, L]} U(x-y) \varphi_{\eta}(x) \varphi_{\eta}(y)
$$

Lemma 2. The function $h_{\kappa}^{L}$ is continuous and uniformly converges to the function $h_{\kappa}$ when $L$ tends to infinity.

The proof of Lemma 2 will be given later.

By using the definition of the $h_{\kappa}^{L}$ we get

$$
\begin{aligned}
\mathbf{Q} & =\left(h_{\kappa}^{L}-h_{\kappa_{0}}\right)|\operatorname{supp} K|-\mu\left(\kappa-\kappa_{0}\right)|\operatorname{supp} K| \\
& =\left(h_{\kappa}^{L}-h_{\kappa_{0}}-\mu\left(\kappa-\kappa_{0}\right)\right)|\operatorname{supp} K|
\end{aligned}
$$

Now let us consider the expression $h_{\kappa}-h_{\kappa_{0}}-\mu\left(\kappa-\kappa_{0}\right)$. According to Theorem 2, the function $h_{\kappa}$ is convex and has a right-hand derivative $\mu_{\kappa}^{+}$ at the point $\kappa$. Therefore

$$
h_{\kappa}-h_{\kappa_{0}} \geqslant \mu_{\kappa}^{+}\left(\kappa-\kappa_{0}\right) \geqslant \mu\left(\kappa-\kappa_{0}\right)
$$

The last inequality is valid due to the assumption $\mu \in\left(\mu_{\kappa}^{-}, \mu_{\kappa}^{+}\right)$(note that $\kappa-\kappa_{0}$ is positive and greater than unity). 
Let

$$
\varepsilon_{1}=\mu_{\kappa}\left(\kappa_{0}-\kappa\right)-\left(h_{\kappa_{0}}-h_{\kappa}\right)
$$

According to Lemma 2, there exists $L_{1}$ such that if $L>L_{1}$, then

$$
h_{\kappa}-h_{\kappa}^{L}>\varepsilon_{1}
$$

Finally, the formulas (23)-(28) lead to the required estimation with

$$
t=t_{\eta}=\left(\mu_{\kappa}\left(\kappa_{0}-\kappa\right)-\left(h_{\kappa_{0}}-h_{\kappa}\right)\right) / 2
$$

Therefore, taking into account the condition $\eta \leqslant n_{0}-1$, we see that in Case $2 \mathrm{~d}$ the required estimation holds at

$$
t=t_{4}=\min _{\eta \leqslant n_{0}-1} t_{\eta}=\left(h_{\left(\kappa_{0}^{-1}-1\right)^{-1}}-h_{\kappa_{0}}-\mu\left(\left(\kappa_{0}^{-1}-1\right)^{-1}-\kappa_{0}\right)\right) / 2
$$

The proof of Case $2 \mathrm{~d}$ is completed.

Case 2e. $n_{0}-1<\eta<n_{0}-1+\delta$. The density of the configuration $\varphi^{\prime}(x)$ is $\kappa=\eta^{-1}$. If $n_{0}+1 \leqslant \eta$ (Case 2d), then [see (25)-(29)]

$$
\begin{aligned}
H\left(\varphi^{\prime}(x)\right)-H\left(\varphi_{n_{0}}(x)\right) & \geqslant \mathbf{Q} \\
& =\left(h_{\kappa}^{L}-h_{\kappa_{0}}-\mu\left(\kappa-\kappa_{0}\right)\right)|\operatorname{supp} K| \\
& \geqslant t_{4}|\operatorname{supp} K|
\end{aligned}
$$

According to Lemma 2, the truncated function of the mean energy $h_{\kappa}^{L}$ is continuous. Therefore, the function $h_{\kappa}^{L}-h_{\kappa_{0}}-\mu\left(\kappa-\kappa_{0}\right)$ is continuous in particular at the point $\left(\kappa_{0}^{-1}-1\right)^{-1}$. Then there exists $\delta_{1}>0$ such that for all $\kappa:\left|\kappa-\left(\kappa_{0}^{-1}-1\right)^{-1}\right|<\delta_{1}$

$$
\left(h_{\kappa}-h_{\kappa_{0}}-\mu\left(\kappa-\kappa_{0}\right)\right)|\operatorname{supp} K| \geqslant t_{3} / 2|\operatorname{supp} K|
$$

Finally, $\delta=\delta_{1}, t_{5}=t_{4} / 2$.

The proof of Case $2 \mathrm{e}$ is completed.

Case 2f. $n_{0}-1+\delta \leqslant \eta<n_{0}$. The density of the configuration $\varphi^{\prime}(x)$ is $\kappa=\eta^{-1}$. Similarly to Case $2 \mathrm{~d}$, we get [see (15)-(17)]

$$
\begin{aligned}
H\left(\varphi^{\prime}(x)\right)-H\left(\varphi_{n_{0}}(x)\right) & =\mathbf{A}_{1}+\mathbf{B}_{1}+\mathbf{C}_{1} \\
\mathbf{A}_{1}+\mathbf{B}_{1}+\mathbf{C}_{1} & =\left(\mathbf{A}_{1}+\mathbf{B}_{1}-\mathbf{A}_{1}^{\prime}-\mathbf{B}_{1}^{\prime}\right)+\mathbf{A}_{1}^{\prime}+\mathbf{B}_{1}^{\prime}+\mathbf{C}_{1}^{\prime}
\end{aligned}
$$

\section{Lemma 3.}

$$
\mathbf{A}_{1}^{\prime}+\mathbf{B}_{1}^{\prime}+\mathbf{C}_{1}^{\prime} \geqslant 0
$$


The proof of Lemma 3 will be given later.

Let us consider the expression $\left(\mathbf{A}_{1}+\mathbf{B}_{1}-\mathbf{A}_{1}^{\prime}-\mathbf{B}_{1}^{\prime}\right)$.

If the length of the support of a contour is $|\operatorname{supp} K|=L$, then, according to the definition of a contour, the contour $K$ contains at most $d_{0}\left(L+n_{0}\right):\left(d_{0}+1\right) n_{0}$ number of blocks

$$
\varphi_{0}=\underbrace{0 \ldots 01}_{n_{0}}
$$

Then the convexity of $U(x)^{(2)}$ directly yields the estimation

$$
\mathbf{A}_{1}+\mathbf{B}_{1}-\mathbf{A}_{1}^{\prime}-\mathbf{B}_{1}^{\prime} \geqslant t_{\eta} \theta|\operatorname{supp} K|
$$

where

$$
\begin{aligned}
t_{\eta} & =1 / 2\left(1+\eta-n_{0}\right)\left(U\left(n_{0}-1\right)+U\left(n_{0}+1\right)-2 U\left(n_{0}\right)\right) \theta \\
\theta & =\left(L-d_{0} n_{0}\right): 2 L\left(d_{0}+1\right) n_{0}
\end{aligned}
$$

Finally, the formulas (31)-(35) yield the inequality (6) with $t=t_{\eta}$.

Therefore, taking into account the condition $n_{0}-1+\delta \leqslant \eta<n_{0}$, we see that in Case $2 \mathrm{f}$ the required estimation (6) holds at

$$
\begin{aligned}
t & =t_{6}=\min _{\eta \in\left(n_{0}, n_{0}+1-\delta\right]}\left(1+\eta-n_{0}\right)\left(U\left(n_{0}-1\right)+U\left(n_{0}+1\right)-2 U\left(n_{0}\right)\right) \theta \\
& =1 / 2\left(U\left(n_{0}-1\right)+U\left(n_{0}+1\right)-2 U\left(n_{0}\right)\right) \delta \theta
\end{aligned}
$$

For the completing of the proof of Lemma 1 we only have to choose

$$
t^{\prime}=\min _{i=0-6} t_{i}
$$

So the inequality (6) holds at $t=t^{\prime}$ if $d_{0} n_{0}>L_{1}$ (see Definition 2 and the choosing of the $L_{1}$ in the Case $2 \mathrm{~d}$ ).

Lemma 1 is proved.

Note that (see the definition of $\theta$ )

$$
t=t\left(d_{0}\right) \sim \text { const } / d_{0} n_{0}
$$

Remark 1. We could prove the statement of Case 2a by using Lemma 2 (as in Case 2d), but in Case 2a we dispensed with Lemma 2 by using a trick.

Proof of Lemma 2. For each given $\varepsilon$ we have to find $L_{\varepsilon}$ such that if $L>L_{\varepsilon}$, then

$$
\left|h_{\kappa}-h_{\kappa}^{L}\right|<\varepsilon
$$


1. Obviously,

$$
\left|h_{\kappa^{\prime \prime}}^{L}-h_{\kappa}^{L}\right| \leqslant\left|h_{\kappa}-h_{\kappa^{\prime \prime}}\right|
$$

The function $h_{\kappa}$ is continuous. ${ }^{(2)}$ Therefore the function $h_{\kappa}^{L}$ is also continuous.

2. The function $h_{\kappa}$ is continuous. Therefore for each given $\varepsilon / 3$ there exists $\delta$ such that $\left|h_{\kappa}-h_{\kappa^{\prime}}\right|<\varepsilon / 2$ if $\left|\kappa-\kappa^{\prime}\right|<\delta$.

3. Let $\left|h_{\kappa}-h_{\kappa^{\prime \prime}}\right|<\delta$ and $\kappa^{\prime \prime}=q / p$. Let consider the restriction of the special ground state $\varphi_{\kappa^{\prime \prime}}$ on the segment $[-l p, l p]$, where $l$ is some natural number. Obviously, for each given $\varepsilon / 3$ there exists $l$ such that $\left|h_{\kappa^{\prime \prime}}^{L}-h_{\kappa^{\prime \prime}}\right|<\varepsilon / 2$ if $L>l p$.

Now note that

$$
\left|h_{\kappa}-h_{\kappa}^{L}\right| \leqslant\left|h_{\kappa}-h_{\kappa^{\prime \prime}}\right|+\left|h_{\kappa^{\prime \prime}}-h_{\kappa^{\prime \prime}}^{L}\right|+\left|h_{\kappa^{\prime \prime}}^{L}-h_{\kappa}^{L}\right| \leqslant \varepsilon / 3+\varepsilon / 3+\varepsilon / 3=\varepsilon
$$

if $L>l p$.

Hence, Lemma 2 is proved.

Proof of Lemma 3. Let the configuration $\varphi(x)$ with a unique contour $K$ be obtained from the special ground state with a density $\kappa$ by some finite perturbation, and let the configuration $\varphi^{M}(x)$ be a periodic configuration with a period $M$, and with the unique contour $K$ on the period [so the density of the configuration $\varphi^{M}(x)$ is equal to $\kappa^{\prime}$ ]. Then

$$
\begin{aligned}
\mathbf{F}(\mathbf{M})= & \sum_{x, y \in \mathbf{Z}^{1}, x>y ; x \text { or } y \in \operatorname{supp} K} U(x-y)\left(\varphi_{M}(x) \varphi_{M}(y)-\varphi_{n_{0}}(x) \varphi_{n_{0}}(y)\right) \\
& -\mu \sum_{x, y \in \mathbf{Z}^{1}, x \in \operatorname{supp} K}\left(\varphi_{M}(x)-\varphi_{n_{0}}(x)\right) \\
\geqslant & \sum_{x, y \in \mathbf{Z}^{1}, x>y ; x \text { or } y \in[-M, M]} U(x-y)\left(\varphi_{\kappa^{\prime}}(x) \varphi_{\kappa^{\prime}}(y)-\varphi_{n_{0}}(x) \varphi_{n_{0}}(y)\right) \\
& -\mu \sum_{x \in \mathbf{Z}^{1}, x \in[-M, M]}\left(\varphi_{\kappa^{\prime}}(x)-\varphi_{n_{0}}(x)\right)=\mathbf{Q}_{\mathbf{M}}
\end{aligned}
$$

By using the definition of the mean energy we get $\left(\kappa^{\prime}=\eta^{\prime-1}\right.$, $\kappa_{0}=n_{0}^{-1}=q(p)$

$$
\mathbf{Q}_{\mathbf{M}}=\left(h_{\kappa^{\prime}}-h_{\kappa_{0}}\right) 2 M-\mu\left(\kappa^{\prime}-\kappa_{0}\right) 2 M=\left(h_{\kappa^{\prime}}-h_{\kappa_{0}}-\mu\left(\kappa^{\prime}-\kappa_{0}\right)\right) 2 M
$$

Let us consider the expression $h_{\kappa^{\prime}}-h_{\kappa_{0}}-\mu\left(\kappa^{\prime}-\kappa_{0}\right)$. According to Theorem 2, the function $h_{\kappa}$ is convex and has a right-hand derivative $\mu_{\kappa}^{+}$ at the point $\kappa$. Therefore,

$$
h_{\kappa^{\prime}}-h_{\kappa_{0}} \geqslant \mu_{\kappa}^{+}\left(\kappa^{\prime}-\kappa_{0}\right) \geqslant \mu\left(\kappa^{\prime}-\kappa_{0}\right)
$$


The last inequality is valid due to the assumption $\mu \in\left(\mu_{\kappa}^{-}, \mu_{\kappa}^{+}\right)$.

Therefore

$$
\mathbf{F}(\mathbf{M}) \geqslant \mathbf{Q}_{\mathbf{M}} \geqslant 0
$$

Now note that

$$
\begin{aligned}
\mathbf{A}_{1}^{\prime}+ & \mathbf{B}_{1}^{\prime}+\mathbf{C}_{1}^{\prime} \\
= & \sum_{x, y \in \mathbf{Z}^{1}, x>} \sum_{y ; x \text { or } y \in \operatorname{supp} K} U(x-y)\left(\varphi(x) \varphi(y)-\varphi_{n_{0}}(x) \varphi_{n_{0}}(y)\right) \\
& -\mu \sum_{x, y \in \mathbf{Z}^{1}, x \in \operatorname{supp} K}\left(\varphi(x)-\varphi_{n_{0}}(x)\right) \\
= & \lim _{M \rightarrow \infty} F(M) \geqslant 0
\end{aligned}
$$

Lemma 3 is proved.

Remark 2. Lemma 1 shows that a special ground state is a ground state. Below we shall prove that a special ground state is a stable (in the sense of Peierls) ground state.

Now we define and investigate the notion of the interaction between contours.

The contour model corresponding to the formulas (4) and (5) is an interacting contour model since the potential is long range. Below we improve the definition of a contour in order to estimate an interaction. A similar approach was proposed in ref. 15. This method was also used in ref. 16.

Now we estimate the interaction between two contours $K_{i}$ and $K_{j}$, the interaction between two interface contours $I K_{i}$ and $I K_{j}$, and the interaction between contour $K_{i}$ and interface contour $I K_{j}$.

Suppose $\operatorname{supp} K_{l}=\left[a_{l}, b_{l}\right]$ and supp $I K_{l}=\left[a_{l}, b_{l}\right]$. Let

$$
\operatorname{supp} I K_{i}^{+}=\left[b_{i}, a_{i+1}\right] \quad \text { and } \operatorname{supp} I K_{i}^{--}=\left[b_{i-1}, a_{i}\right]
$$

where $b_{0}=p$ if there exists $K \in P B\left(\varphi^{\prime}(x)\right)$ such that $\operatorname{supp} K=[-\infty, p]$ and $b_{0}=-\infty$ otherwise; and $a_{m+1}=q$ if there exists $K \in P B\left(\varphi^{\prime}(x)\right)$ such that $\operatorname{supp} K=[q, \infty]$ and $a_{m+1}=\infty$ otherwise.

1. The contour $K_{i} \in P B\left(\varphi^{\prime}(x)\right)$ interacts with the contour $K_{j} \in P B\left(\varphi^{\prime}(x)\right)$ through all pairs $(x, y)$ such that $(x, y) \in \operatorname{Int}\left(K_{i}, K_{j}\right)$ and $f^{\prime}(x, y) \neq 0$, where

$$
\operatorname{Int}\left(K_{i}, K_{j}\right)=\left[(x, y): x, y \in \mathbf{Z}^{1} ; x \in \operatorname{supp} K_{i}, y \in \operatorname{supp} K_{j}\right]
$$


The value of the interaction is

$$
\begin{aligned}
f^{\prime}(x, y)= & U(x-y)\left(\varphi^{\prime}(x) \varphi^{\prime}(y)\right. \\
& -\psi_{K_{i}}(x) \psi_{K_{i}}(y)+\bar{\varphi}_{\kappa}^{i}(x) \bar{\varphi}_{\kappa}^{i}(y) \\
& \left.-\psi_{K_{j}}(x) \psi_{K_{j}}(y)+\bar{\varphi}_{\kappa}^{j}(x) \bar{\varphi}_{\kappa}^{j}(y)\right)
\end{aligned}
$$

2. The interface contour $I K_{i} \in P B\left(\varphi^{\prime}(x)\right)$ interacts with the interface contour $I K_{j} \in P B\left(\varphi^{\prime}(x)\right.$ ) (let $\left.a_{j}>b_{i}\right)$ through all pairs $(x, y)$ such that $(x, y) \in \operatorname{Int}\left(I K_{i}, I K_{j}\right)$ and $f^{\prime \prime}(x, y) \neq 0$, where

$$
\begin{aligned}
\operatorname{Int}\left(I K_{i}, I K_{j}\right)= & \operatorname{Int}^{1}\left(I K_{i}, I K_{j}\right)+\operatorname{Int}^{2}\left(I K_{i}, I K_{j}\right) \\
& +\operatorname{Int}^{3}\left(I K_{i}, I K_{j}\right)+\operatorname{Int}^{4}\left(I K_{i}, I K_{j}\right)
\end{aligned}
$$

$\operatorname{Int}^{1}\left(I K_{i}, I K_{j}\right)=\left[(x, y): x, y \in \mathbf{Z}^{1} ; x \in \operatorname{supp} I K_{i}\right.$ and $\left.y \in \operatorname{supp} I K_{j}\right]$

$\operatorname{Int}^{2}\left(I K_{i}, I K_{j}\right)=\left[(x, y): x, y \in \mathbf{Z}^{1} ; x \in \operatorname{supp} I K_{i}\right.$ and $\left.y \in \operatorname{supp} I K_{j}^{+}\right]$

$\operatorname{Int}^{3}\left(I K_{i}, I K_{j}\right)=\left[(x, y): x, y \in \mathbf{Z}^{1} ; x \in \operatorname{supp} I K_{i}^{-}\right.$and $\left.y \in \operatorname{supp} I K_{j}\right]$

$\operatorname{Int}^{4}\left(I K_{i}, I K_{j}\right)=\left[(x, y): x, y \in \mathbf{Z}^{1} ; x \in \operatorname{supp} I K_{i}^{-}\right.$and $\left.y \in \operatorname{supp} I K_{j}^{+}\right]$

The value of the interaction is

$$
\begin{aligned}
f^{\prime \prime}(x, y)=f_{1}^{\prime \prime}(x, y)= & U(x-y)\left(\varphi^{\prime}(x) \varphi^{\prime}(y)\right. \\
& -\psi_{I K_{i}}(x) \psi_{I K_{i}}(y)+\bar{\varphi}_{\kappa}^{i}(x) \bar{\varphi}_{\kappa}^{i}(y) \\
& \left.-\psi_{I K_{j}}(x) \psi_{I K_{j}}(y)+\bar{\varphi}_{\kappa}^{j}(x) \bar{\varphi}_{\kappa}^{j}(y)\right)
\end{aligned}
$$

if $(x, y) \in \operatorname{Int}^{1}\left(I K_{i}, I K_{j}\right)$;

$$
\begin{aligned}
f^{\prime \prime}(x, y)=f_{2}^{\prime \prime}(x, y)= & U(x-y)\left(\varphi^{\prime}(x) \varphi^{\prime}(y)\right. \\
& \left.-\psi_{I K_{i}}(x) \psi_{I K_{i}}(y)+\bar{\varphi}_{\kappa}^{i}(x) \bar{\varphi}_{\kappa}^{i}(y)\right)
\end{aligned}
$$

if $(x, y) \in \operatorname{Int}^{2}\left(I K_{i}, I K_{j}\right)$;

$$
\begin{aligned}
f^{\prime \prime}(x, y)=f_{3}^{\prime \prime}(x, y)= & U(x-y)\left(\varphi^{\prime}(x) \varphi^{\prime}(y)\right. \\
& \left.\left.-\psi_{I K_{j}}(x) \psi_{I K_{j}}(y)\right)+\bar{\varphi}_{\kappa}^{j}(x) \bar{\varphi}_{\kappa}^{j}(y)\right)
\end{aligned}
$$

if $(x, y) \in \operatorname{Int}^{3}\left(I K_{i}, I K_{j}\right)$; and

$$
\begin{aligned}
f^{\prime \prime}(x, y)=f_{4}^{\prime \prime}(x, y)= & U(x-y)\left(\varphi^{\prime}(x) \varphi^{\prime}(y)\right. \\
& \left.-\bar{\varphi}_{\kappa}^{1, i}(x) \bar{\varphi}_{\kappa}^{1, i}(y)-\bar{\varphi}_{\kappa}^{2, j}(x) \bar{\varphi}_{\kappa}^{2, j}(y)\right)
\end{aligned}
$$

if $(x, y) \in \operatorname{Int}^{4}\left(I K_{i}, I K_{j}\right)$ [the configuration $\bar{\varphi}_{\kappa}^{1, i}(x)$ is defined in Definition 3]. 
3. The contour $K_{i} \in P B\left(\varphi^{\prime}(x)\right)$ interacts with the interface contour $I K_{j} \in P B\left(\varphi^{\prime}(x)\right)$ through all pairs $(x, y)$ such that $(x, y) \in \operatorname{Int}\left(K_{i}, I K_{j}\right)$ and $f^{\prime \prime \prime}(x, y) \neq 0$, where

$$
\begin{aligned}
\operatorname{Int}\left(K_{i}, I K_{j}\right) & =\operatorname{Int}^{1}\left(K_{i}, I K_{j}\right)+\operatorname{Int}^{2}\left(K_{i}, I K_{j}\right) \\
\operatorname{Int}^{1}\left(K_{i}, I K_{j}\right) & =\left[(x, y): x, y \in \mathbf{Z}^{1} ; x \in \operatorname{supp} K_{i} \text { and } y \in \operatorname{supp} I K_{j}\right] \\
\operatorname{Int}^{2}\left(K_{i}, I K_{j}\right) & =\left[(x, y): x, y \in \mathbf{Z}^{1} ; x \in \operatorname{supp} K_{i} \text { and } y \in \operatorname{supp} I K_{j}^{+}\right]
\end{aligned}
$$

if $a_{j}>b_{i}$, and

$\operatorname{Int}^{2}\left(K_{i}, I K_{j}\right)=\left[(x, y): x, y \in \mathbf{Z}^{1} ; x \in \operatorname{supp} K_{i}\right.$ and $\left.y \in \operatorname{supp} I K_{j}^{-}\right]$

if $a_{i}>b_{j}$.

The value of the interaction is

$$
\begin{aligned}
f^{\prime \prime \prime}(x, y)=f_{1}^{\prime \prime \prime}(x, y)= & U(x-y)\left(\varphi^{\prime}(x) \varphi^{\prime}(y)\right. \\
& -\psi_{K_{i}}(x) \psi_{K_{i}}(y)+\bar{\varphi}_{\kappa}^{i}(x) \bar{\varphi}_{\kappa}^{i}(y) \\
& \left.\left.-\psi_{I K_{j}}(x) \psi_{I K_{j}}(y)\right)+\bar{\varphi}_{\kappa}^{j}(x) \bar{\varphi}_{\kappa}^{j}(y)\right)
\end{aligned}
$$

if $(x, y) \in \operatorname{Int}^{1}\left(K_{i}, I K_{j}\right)$, and

$$
\begin{aligned}
f^{\prime \prime \prime}(x, y) & =f_{2}^{\prime \prime \prime}(x, y) \\
& =U(x-y)\left(\varphi^{\prime}(x) \varphi^{\prime}(y)-\psi_{K_{i}}(x) \psi_{K_{i}}(y)+\bar{\varphi}_{\kappa}^{i}(x) \bar{\varphi}_{\kappa}^{i}(y)\right)
\end{aligned}
$$

if $(x, y) \in \operatorname{Int}^{2}\left(K_{i}, I K_{j}\right)$.

By using the formulas (42)-(48) later we shall introduce and investigate the noninteracting contour model.

The following lemma will be extremely useful for the further calculations.

Lemma 4. 1. Let us consider two contours (usual or interface), say $K_{i}$ and $K_{j}$. Suppose $\operatorname{supp} K_{i}=\left[a_{i}, b_{i}\right]$, supp $K_{j}=\left[a_{j}, b_{j}\right]$, or supp $K_{j}=$ $\left[a_{j},+\infty\right]$ and the distance between $\operatorname{supp} K_{i}$ and $\operatorname{supp} K_{j}$ is $a_{j}-b_{i}=$ $R_{i, j}>0$. Let $\operatorname{supp} K_{i}=\operatorname{supp}^{1} K_{i} \cup \operatorname{supp}^{2} K_{i}$ and $\operatorname{supp} K_{j}=\operatorname{supp}^{1} K_{j} \cup$ $\operatorname{supp}^{2} K_{j}$ (see the definition of the essential and regular parts of the support of a contour), and $N=\min \left(\left|\operatorname{supp}^{1} K_{i}\right|,\left|\operatorname{supp}^{1} K_{j}\right|\right)$. Then

$$
\left|G\left(K_{i}, K_{j}\right)\right|=\sum_{(x, y) \in \operatorname{Int}\left(K_{i}, K_{j}\right)}|f(x, y)| \leqslant C_{1} R_{i, j}^{-\alpha} N^{1-\alpha}
$$

where $C_{1}=120 A n_{0}$. 
Proof. Part A. Let us consider two intervals $[a, b]$ and $[c, \infty)$ such that $b-a=N_{1}$, and $c-b=R>0$. Consider the following expression:

$$
G(a, b, c)=\sum_{x, y \in \mathbf{Z}^{1} ; y \in[a, b] \text { and } x \in[c, \infty]} 5 U(x-y)
$$

Then

$$
G(a, b, c) \leqslant C_{1} R^{-\alpha} N_{1}^{1-\alpha} ; \quad C_{1}=30 A
$$

In fact,

$$
\begin{aligned}
G(a, b, c) & =\sum_{i=R}^{R+N_{1}} U(i)(i-R+1)+\left(N_{1}+1\right) \sum_{j=R+N_{1}+1}^{\infty} U(j) \\
& =\mathbf{S}_{1}+\mathbf{S}_{2} \\
\mathbf{S}_{1} \leqslant \mathbf{S}_{1}^{\prime} & =U(R)+\int_{1}^{N_{1}} U(R+z)(z+1) d z \\
& =U(R)+\int_{1}^{N_{1}} U(R+z)(R+z) d z-\int_{1}^{N_{1}} U(R+z)(R-1) d z \\
& =U(R)+\mathbf{I}_{1}-\mathbf{I}_{2}
\end{aligned}
$$

By using the condition $U(x) \sim A x^{-1-\alpha}, 0<\alpha<1$, we have $(R \rightarrow \infty)$

$$
\begin{aligned}
U(R) & \sim A R^{-1-\alpha} \\
\mathbf{I}_{1} & \sim A(1-\alpha)^{-1}\left(\left(R+N_{1}\right)^{1-\alpha}-(R+1)^{(1-\alpha)}\right) \sim A N_{1}^{1-\alpha} R^{-\alpha} \\
\mathbf{I}_{2} & \sim A \alpha^{-1}(R-1)\left((R+1)^{-\alpha}-\left(R+N_{1}\right)^{-\alpha}\right) \sim A N_{1}^{1-\alpha} R^{-\alpha}(R-1) / R
\end{aligned}
$$

Therefore,

$$
\mathbf{I}_{1}-\mathbf{I}_{2} \sim A N_{1}^{1-\alpha} R^{-\alpha}(1-(R-1) / R) \sim A N_{1}^{1-\alpha} R^{-1-\alpha}
$$

Finally, from (50)-(54) we get

$$
\mathbf{S}_{1} \leqslant \mathbf{S}_{1}^{\prime} \leqslant C_{1} R^{-1-\alpha} N_{1}^{1-\alpha} ; \quad C_{1}=3 A
$$

Now note that

$$
\begin{aligned}
\mathbf{S}_{2} & =\left(N_{1}+1\right) \sum_{j=R+N_{1}+1}^{\infty} U(j) \leqslant \mathbf{S}_{2}^{\prime} \\
& =\left(N_{1}+1\right) U\left(R+N_{1}+1\right)+\left(N_{1}+1\right) \int_{z=R+N_{1}+1}^{\infty} U(z) d z \\
& =\left(N_{1}+1\right) U\left(R+N_{1}+1\right)+\mathbf{I}_{2}
\end{aligned}
$$


By using the condition $U(x) \sim A x^{-1-x}, 0<\alpha<1$, we have $(R \rightarrow \infty)$

$$
\begin{aligned}
&\left(N_{1}+1\right) U\left(R+N_{1}+1\right) \sim A R^{-1-\alpha} N_{1}^{-\alpha} \\
& \mathbf{I}_{2} \sim A \alpha\left(N_{1}+1\right)\left(R+N_{1}+1\right)^{-\alpha} \sim A \alpha R^{-\alpha} N_{1}^{1-\alpha}
\end{aligned}
$$

Finally, from (56)-(58) we get

$$
\mathbf{S}_{2} \leqslant C_{1} R^{-\alpha} N_{1}^{1-x} ; \quad C_{1}=3 A
$$

Finally, the inequalities (55) and (59) yield the required inequality (51).

Part B. Let us consider two segments $[a, b]$ and $[c, \infty)$ such that $b-a=M_{1}$ and $c-b=R>0$. Suppose that $K$ is an arbitrary contour with $\operatorname{supp} K=[c, \infty) ; \psi_{a, b}^{1}$ and $\psi_{a, b}^{2}$ are two regular phases. Consider the following expression:

$$
G^{r}(a, b, c)=\sum_{x, y \in \mathbf{Z}^{1} ; x \in[a, b] \text { and } y \in[c, \infty)} 5 U(x-y)\left(\psi_{a, b}^{1}(x) \varphi(y)-\psi_{a, b}^{2}(x) \varphi(y)\right)
$$

Then

$$
G^{r}(a, b, c) \leqslant C_{1} R^{-1-\alpha} ; \quad C_{1}=30 A n_{0}
$$

First, note that there exists a number $p^{\prime}<n_{0}$ such that $\psi_{a, b}^{1}(x)=$ $\psi_{a, b}^{1}(x+p)$. Therefore

$$
\begin{aligned}
& U(x-y)\left(\psi_{a, b}^{1}(x) \varphi(y)-\psi_{a, b}^{2}(x) \varphi(y)\right) \\
& \quad \leqslant\left(U(y-x)-U(y-x+p) \leqslant C_{0} U(x-y) /(x-y)\right.
\end{aligned}
$$

where $C_{0}=\alpha p^{\prime}<\alpha n_{0}$. Then

$$
\begin{aligned}
1 / C_{0} G^{r}(a, b, c) \leqslant & \sum_{x, y \in \mathbf{Z}^{1} ; x \in[a, b] \text { and } y \in[c, \infty)} U(x-y) /(x-y) \\
= & \sum_{i=R}^{R+N_{1}}(i-R+1) U(i) / i+\left(M_{1}+1\right) \cdot \sum_{j=R+M_{1}+1}^{\infty} U(j) / j \\
= & \mathbf{S}_{1}^{r}+\mathbf{S}_{2}^{r} \\
\mathbf{S}_{1}^{r} \leqslant & U(R) / R+\int_{1}^{M_{1}}(z+1) U(R+z) /(R+z) d z \\
= & U(R) / R+\int_{1}^{M_{1}} U(R+z) d z \\
& -\int_{1}^{M_{1}}(R-1) U(R+z) /(R+z) d z \\
= & U(R) / R+\mathbf{I}_{1}^{r}-\mathbf{I}_{2}^{r}
\end{aligned}
$$


By employing the condition $U(x) \sim A x^{-1-\alpha}, 0<\alpha<1$, we have $(R \rightarrow \infty)$

$$
\begin{aligned}
U(R) / R & \sim A R^{-2-\alpha} \\
\mathbf{I}_{1}^{r} & \sim A \alpha^{-1}\left((R+1)^{-\alpha}-\left(R+M_{1}\right)^{-\alpha}\right) \\
\mathbf{I}_{2}^{r} & \sim A(1+\alpha)^{-1}(R-1)\left((R+1)^{-1-\alpha}-\left(R+M_{1}\right)^{-1-\alpha}\right)
\end{aligned}
$$

Therefore,

$$
\mathbf{I}_{1}^{r}-\mathbf{I}_{2}^{r} \sim A R^{-1-\alpha}
$$

Finally, from (62)-(64) we get

$$
\mathbf{S}_{1}^{r} \leqslant C_{1} R^{-1-\alpha} ; \quad C_{1}=3 A
$$

Now note that

$$
\begin{aligned}
\mathbf{S}_{2}^{r}= & \left(M_{1}+1\right) \sum_{j=R+M_{1}+1}^{\infty} U(j) / j \\
\leqslant & \left(M_{1}+1\right) U\left(R+M_{1}+1\right) /\left(R+M_{1}+1\right) \\
& +\left(M_{1}+1\right) \int_{z=R+M_{1}+1}^{\infty} U(z) / z d z \\
= & \left(M_{1}+1\right) U\left(R+M_{1}+1\right) /\left(R+M_{1}+1\right)+\mathbf{I}_{2}^{r}
\end{aligned}
$$

By using the condition $U(x) \sim A x^{-1-\alpha}, 0<\alpha<1$, we have $(R \rightarrow \infty)$

$$
\begin{array}{r}
\left(M_{1}+1\right) U\left(R+M_{1}+1\right) /\left(R+M_{1}+1\right) \leqslant A R^{-1-\alpha} \\
\mathbf{I}_{2}^{r} \sim A \alpha\left(M_{1}+1\right)\left(R+M_{1}+1\right)^{-1-\alpha} \leqslant A R^{-1-\alpha}
\end{array}
$$

Finally, from (66)-(68) we get

$$
\mathbf{S}_{2}^{r} \leqslant C_{1} R^{-1-\alpha} ; \quad C_{1}=3 A
$$

Finally, the inequalities (65) and (69) lead to the required inequality (61).

We estimated the interaction between a finite contour and an infinite contour in Part A and the interaction between a finite regular phase and an infinite contour in Part B. Now we start directly to prove the statements of Lemma 4. We divide the proof of the inequality (49) into three cases.

1. Suppose that we have two ordinary contours, say $K_{i}$ and $K_{j}$. 
The interaction between $K_{1}$ and $K_{2}$ can be estimated as [see (42)]

$$
G\left(K_{1}, K_{2}\right)=\sum_{(x, y) \in \operatorname{Int}\left(K_{i}, K_{j}\right)} f^{\prime}(x, y) \leqslant \sum_{(x, y) \in \operatorname{Int}\left(K_{i}, K_{j}\right)} 5 U(x-y)
$$

Let $\operatorname{supp} K_{i}=\operatorname{supp}^{1} K_{i} \cup \operatorname{supp}^{2} K_{i}$, supp $K_{j}=\operatorname{supp}^{1} K_{j} \cup \operatorname{supp}^{2} K_{j}$, and $N=\min \left(\left|\operatorname{supp}^{1} K_{i}\right|,\left|\operatorname{supp}^{1} K_{j}\right|\right)$. The set of all pairs $(x, y) \in \operatorname{Int}\left(K_{i}, K_{j}\right)$ such that $x \in \operatorname{supp}^{1} K_{i}$ and $y \in \operatorname{supp}^{1} K_{j}$ we denote by $E$. Then

$$
G\left(K_{1}, K_{2}\right) \leqslant \sum_{(x, y) \in E} 5 U(x-y)+\sum_{(x, y) \in \operatorname{Int}\left(K_{i}, K_{j}\right) ;(x, y) \notin E} 6 U(x-y)=S_{1}+S_{2}
$$

Now note that

$$
S_{1} \leqslant G(a, b, c)=\sum_{x, y \in \mathbf{Z}^{1} ; y \in[a, b] \text { and } x \in[c, \infty]} 5 U(x-y)
$$

where $b-a=N$ and $c-b=R$. According to the inequality (51),

$$
S_{1} \leqslant C_{1} R^{-\alpha} N^{1-\alpha} ; \quad C_{1}=30 \mathrm{~A}
$$

In addition, because of the inequality (61) we may write

$$
S_{2} \leqslant C_{1} R^{-1-\alpha} ; \quad C_{1}=30 A n_{0}
$$

Hence in the first case the proof of the statement (49) is completed.

2. Suppose that we have one ordinary and one interface contour, say $K_{i}$ and $I K_{j}$.

The interaction between $K_{i}$ and $K_{2}$ can be estimated as [see (47), (48)]:

$$
\begin{aligned}
G\left(K_{i}, I K_{j}\right) & =\sum_{(x, y) \in \operatorname{Int}\left(K_{i}, I K_{j}\right)} f^{\prime \prime \prime}(x, y) \\
& =\sum_{(x, y) \in \operatorname{Int}^{1}\left(K_{i}, I K_{j}\right)} f_{1}^{\prime \prime \prime}(x, y)+\sum_{(x, y) \in \operatorname{Int}^{2}\left(K_{i}, I K_{j}\right)} f_{2}^{\prime \prime \prime}(x, y) \\
& =\sum_{(x, y) \in \operatorname{Int}^{1}\left(K_{i}, I K_{j}\right)} 5 U(x-y)+\sum_{(x, y) \in \operatorname{Int}^{2}\left(K_{i}, I K_{j}\right)} f_{2}^{\prime \prime \prime}(x, y) \\
& =S_{1}+S_{2}
\end{aligned}
$$

As in the first case, we have

$$
S_{1} \leqslant C_{1} R^{-x} N^{1-x} ; \quad C_{1}=30 A
$$

Now note that the formulas (47) and (48) allow us to apply the inequality (61) and to get the following estimation:

$$
S_{2} \leqslant C_{1} R^{-1-\alpha} ; \quad C_{1}=60 A n_{0}
$$


Therefore in the second case the proof of the statement (49) is completed.

3. Suppose that we have two interface contours, say $I K_{i}$ and $I K_{j}$.

The interactiopn between two interface contours $I K_{1}$ and $I K_{2}$ can be estimated as [see (43)-(47)]

$$
\begin{aligned}
G\left(I K_{1}, I K_{2}\right) & =\sum_{(x, y) \in \operatorname{Int}\left(I K_{i}, I K_{j}\right)} f^{\prime \prime}(x, y) \\
& =\sum_{n=1}^{4} \sum_{(x, y) \in \operatorname{Int}^{n}\left(I K_{i}, I K_{j}\right)} f_{n}^{\prime \prime}(x, y) \\
& \leqslant \sum_{(x, y) \in \operatorname{Int}^{1}\left(I K_{i}, I K_{j}\right)} 5 U(x-y)+\sum_{n=1}^{3} \sum_{(x, y) \in \operatorname{Int}^{n}\left(I K_{i}, I K_{j}\right)} f_{n}^{\prime \prime}(x, y) \\
& =S_{1}+S_{2}
\end{aligned}
$$

As in the first case

$$
S_{1} \leqslant C_{1} R^{-\alpha} N^{1-\alpha} ; \quad C_{1}=30 A
$$

Now note that the formulas (43)-(47) allow us to apply the inequality (61) and to get the following estimation:

$$
S_{2} \leqslant C_{1} R^{-1-\alpha} ; \quad C_{1}=90 A n_{0}
$$

The proof of Lemma 4 is completed.

Now we shall prove seven auxiliary lemmas. Suppose that the boundary condition $\bar{\varphi}(x)=[\varphi(x), x \in(-\infty,-V-1] \cup[V+1, \infty)]$ is fixed. The set of all configurations $\varphi(x), x \in[-V, V]$, we denote by $\Phi(V)$. Let $\varphi^{\prime}(x) \in \Phi(V)$, and an ordinary contour $K$ and an interface contour $I K$ belong to $P B\left(\varphi^{\prime}(x)\right)$.

Lemma 5. There exists a positive constant $t$, depending only on the Hamiltonian (1), such that the weight of an ordinary contour $K$ satisfies the following inequality:

$$
\gamma(K)>t\left|\operatorname{supp}^{1} K\right|
$$

where $t$ is the constant defined in Lemma 1 .

Proof. Lemma 5 is a consequence of formula (4) and Lemma 1.

Indeed, let a contour $K$ contain precontours $P K_{i}, i=1, \ldots, m$. This implies that $\operatorname{supp}^{1} K=\bigcup_{i=1}^{m} \operatorname{supp} P K_{i}$. 
The function of interaction $G\left(P K_{1}, \ldots, P K_{m}\right)$ is a decreasing function of the distance between precontours. Therefore

$$
\gamma(K)=\sum_{i=1}^{m} \gamma(P K) G\left(P K_{1}, \ldots, P K_{m}\right)>\gamma(\bar{K})
$$

where the contour $\bar{K}$ is obtained by merging all neighboring precontours (thus $|\operatorname{supp} \bar{K}|=\left|\operatorname{supp}^{1} \bar{K}\right|=\sum_{i=1}^{m}\left|\sum_{i=1}^{m} \gamma(P K)\right|=\left|\operatorname{supp}^{1} K\right|$ ).

But according to Lemma $1, \gamma(K)>t|\operatorname{supp} \bar{K}|$. Therefore,

$$
\gamma(K)>t\left|\operatorname{supp}^{1} K\right|
$$

Lemma 5 is proved.

Let us consider an arbitrary configuration $\varphi^{\prime}(x) \in \Phi(V)$. The boundary of the configuration $\varphi^{\prime}(x)$ includes a finite number of ordinary contours $K_{i}$, $i=1, \ldots, n$, and a finite number of interface contours $I K_{i}, i=n+1, \ldots, n+m$. Let $K_{i}=K_{i}, i=1, \ldots, n$, and $K_{i}=I K_{i}, i=n+1, \ldots, n+m$. The weights of the $K_{i}$ (contours and interface contours) are defined by formulas (4) and (5).

Let $K_{l}$ be an arbitrary contour of the boundary $B\left(\varphi^{\prime}(x)\right)$, $\left|\operatorname{supp}^{1} K_{l}\right|=N_{l} ; \quad K_{0}=\bar{\varphi}\left(K_{0}\right), \quad \operatorname{supp} K_{0}, \quad$ where $K_{0}=(-\infty,-V-1] \cup$ $[V+1, \infty)$; the distance between supp $K_{l}$ and $\operatorname{supp} K_{0}$ is $R_{l}$.

Lemma 6. Suppose $N_{b}>6 C_{1} / t^{1 / \alpha}\left(N_{b}\right.$ is a constant introduced in the definition of a contour). Then

$$
\gamma\left(K_{l}\right) / 6>\left|G\left(K_{l}\right)\right|=\sum_{j=1 ; j \neq l}^{n+m}\left|G\left(K_{l}, K_{j}\right)\right|
$$

Proof. Lemma 6 is a consequence of Lemmas 4 and 5. In fact, according to Lemmas 4 and 5,

$$
\left|G\left(K_{l}\right)\right|<C_{1} N_{b}^{-x} N_{l}^{1-x}<t N_{l} / 6<\gamma\left(K_{l}\right) / 6
$$

if $N_{b}^{\alpha}>6 C_{1} / t$.

Lemma 6 is proved.

Lemma 7. Suppose $R_{l}>6 C_{1} / t^{1 / x}$. Then

$$
\gamma\left(K_{l}\right) / 6>\left|G\left(K_{l}, K_{0}\right)\right|
$$

Proof. Lemma 7 is a consequence of Lemmas 4 and 5. In fact, according to Lemmas 4 and 5 ,

$$
\left|G\left(K_{l}, K_{0}\right)\right|<C_{1} R_{l}^{-\alpha} \bar{N}_{l}^{1-\alpha}<t N_{l} / 6<\gamma\left(K_{l}\right) / 6
$$

if $R_{l}^{\alpha}>6 C_{1} / t$. 
Lemma 7 is proved.

Lemma 8. Suppose $N_{l}>6 C_{1} / t^{1 / \alpha}$. Then

$$
\gamma\left(K_{l}\right) / 6>\left|G\left(K_{l}, K_{0}\right)\right|
$$

Proof. Lemma 8 is a consequence of Lemmas 4 and 5. In fact, according to Lemmas 4 and 5 ,

$$
\left|G\left(K_{l}, K_{0}\right)\right|<C_{1} R_{l}^{-\alpha} N_{l}^{1-\alpha}<t N_{l} / 6<\gamma\left(K_{l}\right) / 6
$$

if $N_{l}^{\alpha}>6 C_{1} / t$.

Lemma 8 is proved.

Suppose $I K$ is an interface contour. Let us consider the configuration $\psi_{I K}$ such that the boundary of the configuration $\psi_{I K}$ includes only the contour $I K$ (see Definition 3). Let the special ground states $\varphi_{n_{0}}^{1}(x)$ and $\varphi_{n_{0}}^{2}(x)$ be defined as in Definition 3. The set of all configurations $\varphi^{\prime}(x) \in \Phi$ such that $P B\left(\varphi^{\prime}(x)\right)$ contains the only interface contour and the same special ground states $\varphi_{n_{0}}^{1}(x)$ and $\varphi_{n_{0}}^{2}(x)$ we denote by $\Phi^{1,2}$.

Lemma 9. Let the configurations $\varphi(x), \varphi^{\prime}(x) \in \Phi^{1,2}$ contain unique interface contours $I K$ and $I K^{\prime}$, respectively, and two regular phases $\psi^{1}(x)$ and $\psi^{2}(x)$. Moreover, let the restriction of the configuration $\varphi^{\prime}(x)$ on supp $I K$ coincide with $\varphi(\operatorname{supp} I K)$ and $\left|\operatorname{supp} I K^{\prime}\right|-|\operatorname{supp} K|>N_{b}$.

There exists a positive constant $t$ depending on the Hamiltonian (1) only, such that the weight of any interface contour $I K$ satisfies the following inequality:

$$
\gamma\left(I K^{\prime}\right)-\gamma(I K)>t\left(\left|\operatorname{supp} I K^{\prime}\right|-|\operatorname{supp} K|-N_{b}\right)
$$

Proof. Let the configurations $\varphi$ and $\varphi^{\prime}$ be obtained, respectively, from the configurations $\varphi(x)$ and $\varphi^{\prime}(x)$ by substitution of $\psi^{2}(x)$ by $\psi^{1}(x)$. In other words, the configurations $\varphi$ and $\varphi^{\prime}$ are obtained by shifting of the regular phase $\psi^{2}(x)$ for some $p<n_{0}$. The configurations $\varphi(x)$ and $\varphi^{\prime}$ contain the usual contours $K$ and $I K$. Then

$$
\begin{aligned}
H(\varphi(x))-H(\varphi) & \leqslant \sum_{x \in \mathbf{Z}^{1} ; x>0}(U(x)-U(x+p)) x \\
& \leqslant \sum_{x \in \mathbf{Z}^{\dagger} ; x>0} 2 A x\left(x^{-\alpha}-(x+p)^{-\alpha}\right) \\
& \leqslant \sum_{x \in \mathbf{Z}^{1}, x>0} 2 A \alpha p x^{-\alpha}<\mathrm{const}
\end{aligned}
$$


and

$$
\begin{aligned}
H\left(\varphi^{\prime}(x)\right)-H\left(\varphi^{\prime}\right) & \leqslant \sum_{x \in \mathbf{Z}^{1} ; x>0}(U(x)-U(x+p)) x \\
& \leqslant \sum_{x \in \mathbf{Z}^{1} ; x>0} 2 A x\left(x^{-\alpha}-(x+p)^{-\alpha}\right) \\
& \leqslant \sum_{x \in \mathbf{Z}^{1} ; x>0} 2 A \alpha p x^{-\alpha}<\mathrm{const}
\end{aligned}
$$

Therefore,

$$
\begin{aligned}
\gamma\left(I K^{\prime}\right)-\gamma(K) & =H\left(\varphi^{\prime}(x)\right)-H\left(\psi^{1}(x)\right)-\left(H(\varphi(x))-H\left(\psi^{1}(x)\right)\right) \\
& =H\left(\varphi^{\prime}(x)\right)-H(\varphi(x))>H\left(\varphi^{\prime}\right)-H(\varphi)-\mathrm{const}
\end{aligned}
$$

Now note that according to Lemma 8

$$
H\left(\varphi^{\prime}(x)\right)-H(\varphi(x))=\gamma\left(K^{\prime}\right)-\gamma(K)>t\left(\left|\operatorname{supp} I K^{\prime}\right|-|\operatorname{supp} K|-N_{b}\right)
$$

Lemma 9 is proved.

Lemma 10. There exists a configuration $\varphi_{\text {min }}^{1,2}(x) \in \Phi^{1,2}$ such that

$$
H\left(\varphi^{1,2}(x)\right)-H(\varphi(x)) \geqslant 0
$$

for each configuration $\varphi(x) \in \Phi^{1,2}$. Let $I K_{\min }^{1,2}$ be a unique contour of the configuration $\varphi_{\text {min }}^{1,2}$. Then $\left|\operatorname{supp} I K_{\min }^{1,2}\right|<3 d_{0} n_{0}+N_{b}$.

Proof. Lemma 10 is a consequence of Lemma 9.

Indeed, let us consider the configuration $\varphi(x) \in \Phi^{1,2}$ with the unique interface contour $K$ such that $|\operatorname{supp} I K|<3 d_{0} n_{0}$ [the existence of the configuration $\varphi(x)$ is obvious]. Then according to Lemma 9

$$
\left|\operatorname{supp} I K_{\text {min }}^{1,2}\right|<|\operatorname{supp} I K|+N_{b}<3 d_{0} n_{0}+N_{b}
$$

Lemma 11. Suppose $\varphi(x), \varphi^{\prime}(x) \in \Phi(V)$ and the restriction of $\varphi(x)$ on some segment $[a, b] \in\left[-V+N_{b}, V-N_{b}\right]$ coincides with a special ground state $\varphi_{n_{0}}^{1}(x)$. The configuration $\varphi^{\prime}(x)$ is some perturbation of $\varphi(x)$ on the segment $[a, b]$. The set $P B\left(\varphi^{\prime}(x) ;[a, b]\right)$ is the set of all contours (usual and interface) $K \in P B\left(\varphi^{\prime}(x)\right)$ and supp $K_{i} \in[a, b]$. Suppose that $P B\left(\varphi \phi^{\prime}(x) ;[a, b]\right)=\bigcup_{i=1}^{n} K_{i}$. Then there exists a positive constant $t$ depending on the Hamiltonian (1) only, such that

$$
\sum_{i=1}^{n} \gamma\left(K_{i}\right)>t \sum_{i=1}^{n}\left|\operatorname{supp}^{1} K_{i}\right|
$$


Proof. The proof coincides with the proof of Lemma 5.

Now we are ready to prove the following result.

Lemma 12. Suppose that the boundary conditions $\bar{\varphi}(x)=[\varphi(x)$, $x \in(-\infty,-V-1] \cup[V+1, \infty)]$ are fixed and $N_{b}>6 C_{1} / t^{1 / \alpha}$. The set of all configurations $\varphi(x), x \in[-V, V]$, we denote by $\Phi(V)$. Let $\varphi_{\min }(x) \in \Phi(K)$ be a configuration with the minimal energy

$$
H\left(\varphi_{\min }(x)\right)=\lim _{\varphi(x) \in \Phi(K)} H(\varphi(x))
$$

Then the configuration $\varphi_{\min }(x)$ has the following structure:

The restriction of the configuration $\varphi_{\min }(x)$ on the set $\left[-V+N_{b}\right.$, $\left.V-N_{b}\right]$ contains at most $n_{0}-1$ contours; moreover, all of them are interface contours $I K_{i}, \quad i=1, \ldots, m$, where $m<n_{0}-1$ and $\left|\operatorname{supp} I K_{i}\right|<$ $3 d_{0} n_{0}+N_{b}$.

Proof. Let the boundary of the configuration $\varphi^{\prime}(x)$ include a finite number of ordinary contours $K_{i}, i=1, \ldots, n$, and a finite number of interface contours $I K_{i}, i=n+1, \ldots, n+m$. The set of all contours of the boundary conditions $\bar{\varphi}(x)$ will be denoted by $K_{0}$.

The following equation is a direct consequence of the formulas (4) and (5):

$$
H\left(\varphi^{\prime}(x)\right)-H\left(\varphi_{n_{0}}(x)\right)=\sum_{i=1}^{n+m} \gamma\left(K_{i}\right)+\sum_{i, j=0 ; i<j}^{n+m} G\left(K_{0}, K_{1}, \ldots, K_{n+m}\right)
$$

where $\varphi_{n_{0}}(x)$ coincides with a special ground state $\bar{\varphi}_{\kappa}^{1}(x)$ (see Definition 3 ) defined for the first from the left contour (ordinary or interface) $K_{i}$, $i=1, \ldots, n+m$, and the multiplier $G\left(K_{0}, K_{1}, \ldots, K_{n+m}\right)$ corresponds to the interaction between contours (ordinary and interface) and with the boundary conditions,

$$
G\left(K_{0}, K_{1}, \ldots, K_{n+m}\right)=\sum_{i, j=0 ; i<j}^{n+m} G\left(K_{i}, K_{j}\right)
$$

Let $K_{i}, i=i_{1}, \ldots, i_{p}$, be an arbitrary ordinary contour of the boundary $B$. Then automatically a distance $R_{i}$ between supp $K_{i}$ and supp $\bar{K}$ is greater than $N_{b}$. Then according to Lemma 6

$$
H\left(\varphi^{\prime}(x)\right)-H\left(\varphi^{i}(x)\right)=\gamma\left(K_{i}\right)-\left|G\left(K_{i}\right)\right| \geqslant 0
$$

where the configuration $\varphi^{i}(x)$ is obtained from the configuration $\varphi^{\prime}(x)$ by deleting of contour $K_{i}$. 
By continuing this procedure we get

$$
H\left(\varphi^{\prime}(x)\right)-H\left(\varphi^{i_{1}, \ldots, i_{p}}(x)\right)>0
$$

where the configuration $\varphi^{i_{1}, \ldots, i_{p}}(x)$ is obtained from the configuration $\varphi^{\prime}(x)$ by deleting of contours $K_{i_{1}}, \ldots, K_{i_{p}}$.

Let $\left[K_{i}, i=i_{p+1}, \ldots, i_{p+q}\right]$ be a set of all interface contours of the boundary $B$, such that the distance $R_{i}$ between supp $K_{i}$ and supp $\bar{K}$ is greater than $N_{b}$.

According to Lemma 11, upon deleting all interface contours except at most $n_{0}-1$ (we have at most $n_{0}$ distinct regular phases which correspond to $n_{0}$ distinct special ground states), we can obtain the configuration

$$
\varphi^{\mathrm{del}}=\varphi^{i_{1}, \ldots, i_{p}, i_{p+1}, \ldots, i_{p+q}, \bar{s}}(x)
$$

such that

$$
H\left(\varphi^{\prime}(x)\right)-H\left(\varphi^{\mathrm{del}}\right)(x)>0
$$

Further, let $K_{1}$ and $K_{2}$ be a contours with $\left|\operatorname{supp} K_{i}\right|>N_{b}, i=1,2$, such that $\operatorname{supp} K_{1}=\left[a_{1}, b_{1}\right] \cup\left[b_{1}+1, c_{1}\right]$ and $\operatorname{supp} K_{2}=\left[c_{2}, a_{2}-1\right] \cup$ $\left[a_{2}, b_{2}\right]$, where $b_{1}=-V+N_{b}$ and $a_{2}=V-N_{b}$.

Then, according to Lemma 8 ,

$$
H\left(\varphi^{\mathrm{del}}\right)-H\left(\varphi^{\mathrm{del}, 1}\right)>0
$$

where the configuration $\varphi^{\text {del, } 1}$ is obtained from the configuration $\varphi^{\text {del }}$ by deleting of contours $K_{1}^{\prime}=\left(\varphi^{\mathrm{del}}\left(\left[b_{1}+1, c_{1}\right],\left[b_{1}+1, c_{1}\right]\right)\right.$ and $K_{2}^{\prime}=$ $\left(\varphi^{\mathrm{del}}\left(\left[c_{2}, a_{2}-1\right],\left[c_{2}, a_{2}-1\right]\right)\right.$.

The inequality (77) shows that a configuration with the minimal energy contains at most $n_{0}-1$ interface contours in the interval $\left[-V+N_{b}, V-N_{b}\right]$. Let supp $I K_{i}$ be an interface contour of the configuration $\varphi_{\min }(x)$. According to Lemmas 10 and 6,

$$
\left|\operatorname{supp} I K_{i}\right|<3 d_{0} n_{0}+N_{b}
$$

Therefore, there exists a configuration $\varphi^{\text {del, } 2}$ such that

$$
H\left(\varphi^{\mathrm{del}, 1}\right)-H\left(\varphi^{\mathrm{del}, 2}\right)>0
$$

where the configuration $\varphi^{\mathrm{del}, 2}$ is obtained from the configuration $\varphi^{\text {del. } 1}$ by chopping of large interface contours (contour $I K_{i}$ is said to be large if $\left.\left|\operatorname{supp} I K_{i}\right|>3 d_{0} n_{0}+N_{b}\right)$.

Lemma 12 is proved. 
By using Lemma 12 we shall give a full description of the set of all ground states.

We know that each special ground state is a ground state (see Remark 2).

Theorem 4. Let the value of the external field $\mu$ of the model (1) belong to the interval $\left(\mu_{\kappa}^{-}, \mu_{\kappa}^{+}\right)$for some number $\kappa=q / p=1 / n_{0}$. Suppose that $\varphi^{\prime}(x)$ is a ground state of the model (1). Then the configuration $\varphi^{\prime}(x)$ contains at most $n_{0}-1$ interface contours $I K_{i}$ such that $\left|\operatorname{supp} I K_{i}\right|<$ $3 d_{0} n_{0}+N_{b}<$ const.

Proof. Theorem 4 is a direct consequence of Lemma 12. Indeed, let $\varphi^{\prime}(x)$ be a ground state of the model (1).

Let us consider an arbitrary segment $[-V, V]$, and a boundary condition $\bar{\varphi}(x)=\left[\varphi^{\prime}(x), x \in(-\infty,-V-1] \cup[V+1, \infty)\right]$. The set of all configurations $\varphi(x), x \in[-V, V]$, we denote by $\Phi(V)$. Suppose a configuration $\varphi_{\min }(x) \in \Phi(V)$ is a configuration with the minimal energy [see (75)].

According to the definition of the ground state, ${ }^{(1)}$ for each $x \in\left[-V+N_{b}, V-N_{b}\right]$

$$
\varphi_{\min }(x)=\varphi^{\prime}(x)
$$

and the configuration $\varphi^{\prime \prime}(x)$ according to Lemma 12 has the required form in the segment $\left[-V+N_{b}, V-N_{b}\right]$. Note that $V$ can be chosen arbitrarily large. The proof of Theorem 4 is completed.

Theorem 4 shows that each ground state is a special ground state or a combination of several special ground states.

\section{GIBBS STATES. THE DENSITY $\mathrm{k}$ IS $1 / n_{0}$}

In this section we prove the uniqueness of the Gibbs states for special values of the external field (when the density of the special ground state is $1 / n_{0}$ ). The general case will be considered in Section 4 .

Suppose that the value of the external field $\mu$ of the model (1) belongs to the interval $\left(\mu_{\kappa}^{-}, \mu_{\kappa}^{+}\right)$for some number $\kappa=q / p=1 / n_{0}$, and the boundary conditions $\bar{\varphi}(x)=[\varphi(x), x \in(-\infty,-V-1] \cup[V+1, \infty)]$ are fixed.

Let $\varphi(x) \in \Phi(V)$ be an arbitrary configuration; the boundary of the $\varphi(x)$ includes a finite number of usual contours $K_{i}, i=1, \ldots, n$, and a finite number of interface contours $I K_{i}, i=n+1, \ldots, n+m$. Let $K_{i}=K_{i}, i=1, \ldots, n$, and $K_{i}=I K_{i}, i=n+1, \ldots, n+m$. The set of all contours of the boundary conditions $\bar{\varphi}(x)$ will be denoted by $K_{0}$. 
The statistical weights of contours and interface contours are

$$
w\left(K_{i}\right)=\exp \left(-\beta \gamma\left(K_{i}\right)\right)
$$

Let

$$
\begin{aligned}
H(\varphi(x) \mid \bar{\varphi}(x))= & -\mu \sum_{x \in \mathbf{Z}^{1}, x \in[-V, V]} \varphi(x) \\
& +\sum_{x, y \in \mathbf{Z}^{1}, x>y ; x, y \in[-V, V]} U(x-y) \varphi(x) \varphi(y) \\
& +\sum_{x, y \in \mathbf{Z}^{1}, x>y ; x \in[-V, V] ; y \notin[-V, V]} U(x-y) \varphi(x) \bar{\varphi}(y) \\
& +\sum_{x, y \in \mathbf{Z}^{1}, x>y ; x \notin[-V, V], y \in[-V, V]} U(x-y) \bar{\varphi}(x) \varphi(y)
\end{aligned}
$$

The following equation is a direct consequence of the formulas (4), (5), and (79):

$$
\exp \{-\beta H(\varphi(x) \mid \bar{\varphi}(x))\}=\prod_{i=1}^{n+m} w\left(K_{i}\right) \exp \left\{-\beta G\left(K_{0}, K_{1}, \ldots, K_{n+m}\right)\right\}
$$

where the multiplier $G\left(K_{0}, K_{1}, \ldots, K_{n+m}\right)$ corresponds to the interaction between contours [usual and interface; see (42)-(48)] and with the boundary conditions $\bar{\varphi}(x)$ :

$$
\begin{aligned}
G\left(K_{0}, K_{1}, \ldots, K_{n+m}\right) & =\sum_{i, j=0 ; i<j}^{n+m} G\left(K_{i}, K_{j}\right) \\
& =\sum_{i, j ; i<j} \sum_{(x, y) \in \operatorname{Int}\left(K_{i}, K_{j}\right)} f(x, y)
\end{aligned}
$$

For simplicity, $K_{i}, i=1, \ldots, n+m$, will be denoted by $K_{i}, i \in \mathbf{I}$, where the statistical weights are defined by the formulas (79), (4), and (5). Thus, the formula (80) has the form

$$
\exp \{-\beta H(\varphi(x) \mid \bar{\varphi}(x))\}=\prod_{i \in \mathbf{I}} w\left(K_{i}\right) \exp \left\{-\beta G\left(K_{0}, K_{1}, \ldots, K_{n+m}\right)\right\}
$$

The set of all pairs $(x, y)$ in the double sum $(81)$ will be denoted by $G$. Write (82) as follows:

$$
\exp \{-\beta H(\varphi(x) \mid \bar{\varphi}(x))\}=\prod_{i \in \mathbf{I}} w\left(K_{i}\right) \prod_{(x, y) \in G}\{1+\exp (-\beta f(x, y)-1)\}
$$

From (83) we get

$$
\exp \{-\beta H(\varphi(x) \mid \bar{\varphi}(x))\}=\sum_{G^{\prime} \in G} \prod_{i \in \mathbf{I}} w\left(K_{i}\right) \prod_{(x, y) \in G^{\prime} ; f(x, y) \neq 0} g(x, y)
$$


where the summation is taken over all subsets $G^{\prime}$ (including the empty set) of the set $G$, and $g(x, y)=\exp (-\beta f(x, y))-1$.

Consider an arbitrary term of the sum (84), which corresponds to the subset $G^{\prime} \subset G$. Let the bond $(x, y) \in G^{\prime}$. Below, contours and interface contours will be called contours. Consider the set $\mathbf{K}$ of all contours such that for each contour $K \subset \mathbf{K}$, the set $\operatorname{supp} K \cap(x \cup y)$ contains one point. We call any two contours from $\mathbf{K}$ connected. The set of contours $K^{\prime}$ is called $G^{\prime}$ connected if for any two contours $K_{p}$ and $K_{q}$ there exists a collection $\left(K_{1}=K_{p}, K_{2}, \ldots, K_{n}=K_{q}\right)$ such that any two contours $K_{i}$ and $K_{i+1}$, $i=1, \ldots, n-1$, are connected by some bond $(x, y) \in G^{\prime}$.

Definition 4. The pair $D=\left[\left(K_{i}, i=1, \ldots, s\right) ; G^{\prime}\right]$, where $G^{\prime}$ is some set of bonds, is called a cluster provided there exists a configuration $\varphi(x)$ such that $K_{i} \in P B(\varphi(x)), i=1, \ldots, s ; G^{\prime} \subset G$; and the set $\left(K_{i}, i=1, \ldots, s\right)$ is $G^{\prime}$ connected. The statistical weight of a cluster $D$ is defined by the formula

$$
w(D)=\prod_{i=1}^{s} w\left(K_{i}\right) \prod_{(x, y) \in G^{\prime}} g(x, y)
$$

Two clusters $D_{1}$ and $D_{2}$ are called compatible provided any two contours $K_{1}$ and $K_{2}$ belonging to $D_{1}$ and $D_{2}$, respectively, are compatible and not connected. A set of clusters is called compatible provided any two clusters of it are compatible.

If $D=\left[\left(K_{i}, i=1, \ldots, s\right) ; G^{\prime}\right]$, then we say that $K_{i} \in D, i=1, \ldots, s$.

Lemma 13. Let boundary conditions $\bar{\varphi}(x)=[\varphi(x), x \in(-\infty,-V-1] \cup$ $[V+1, \infty)]$ be fixed.

If $\left[D_{1}, \ldots, D_{m}\right]$ is a compatible set of clusters and $\bigcup_{i=1}^{m} \operatorname{supp} D_{i} \subset$ $[-V, V]$, then there exists a configuration $\varphi(x)$ which contains this set of clusters. For each configuration $\varphi(x)$ we have

$$
\exp \{-\beta H(\varphi(x) \mid \bar{\varphi}(x))\}=\sum_{G^{\prime} \in G} \prod w\left(D_{i}\right)
$$

where the clusters $D_{i}$ are completely determined by the set $G^{\prime}$. The partition function is

$$
\Xi(\bar{\varphi}(x))=\sum w\left(D_{1}\right) \cdots w\left(D_{m}\right)
$$

where the summation is taken over all nonordered compatible collections of clusters.

Proof. The proof of Lemma 13 follows immediately from the definitions. 


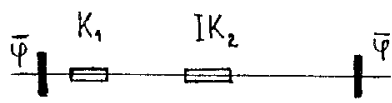

(a)

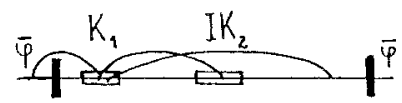

(b)

Fig. 1.

Lemma 13 shows that we come to noninteracting clusters from interacting contours. Figure 1a shows contours $K_{1}$ and $I K_{2}$ of a configuration $\varphi(x)$ and Fig. 1b shows a cluster (one of many) corresponding to these contours.

Lemma 14. Let $D=\left[\left(K_{i}, i=1, \ldots, s\right) ; G^{\prime}\right]$ be an arbitrary cluster. Let $\operatorname{supp}^{1} D=\sum_{i=1}^{s} \operatorname{supp}^{1} K_{i}$ and $t_{1}=5 t / 6$, where $t$ is the constant defined in Lemma 5. Then

$$
w(D)<\exp \left(-\beta t_{1}\left|\operatorname{supp}^{1} D\right|\right)
$$

Proof. This lemma follows from Lemmas 5 and 6. Indeed,

$$
\begin{aligned}
w(D) & =\prod_{i=1}^{s} w\left(K_{i}\right) \prod_{(x, y) \in G^{\prime}} g(x, y) \\
& <\prod_{i=1} \exp \left(-\beta t\left|\operatorname{supp}^{1} K_{i}\right|\right) \prod_{(x, y) \in G^{\prime}} g(x, y) \\
& \leqslant \prod_{i=1}^{s} \exp \left(-\beta t\left|\operatorname{supp}^{1} K_{i}\right|\right) \prod_{(x, y) \in G^{\prime}} \exp (|\beta f(x, y)|) \\
& \leqslant \exp \left\{-\beta \sum_{i=1}^{s} t\left|\operatorname{supp}^{1} K_{i}\right|+\sum_{(x, y) \in G^{\prime}} \beta|f(x, y)|\right\} \\
& <\exp \left(-5 \beta t\left|\operatorname{supp}^{1} D\right| / 6\right)
\end{aligned}
$$

Lemma 14 is proved.

Lemma 15. Suppose that the value of the external field $\mu$ of the model (1) belongs to the interval $\left(\mu_{\kappa}^{-}, \mu_{\kappa}^{+}\right)$for some number $\kappa=q / p=1 / n_{0}$, and boundary conditions $\bar{\varphi}(x)=[\varphi(x), x \in(-\infty,-V-1] \cup[V+1, \infty)]$ are fixed.

In the proof of Lemma 12 we have defined a configuration $\varphi^{\text {del, } 2}$ for each configuration $\varphi(x) \in \Phi(V)$. Let us consider the set of all configurations $\varphi^{\text {del, } 2}(x)$. According to Lemma 12 (a boundary of each configuration 
$\varphi^{\text {del, } 2}(x)$ contains at most $n_{0}-1$ interface contours and two contours around the boundary),

$$
\max \left|B\left(\varphi^{\mathrm{del}, 2}(x)\right)\right| \leqslant\left(3 d_{0} n_{0}+N_{b}\right)\left(n_{0}+1\right)=J_{1}
$$

Let $J=J_{1}+N_{b}\left(n_{0}+1\right)$.

Let $D$ be an arbitrary cluster, such that $|\operatorname{supp} D|>J$.

Then the probability of the cluster $D$ is given by

$$
P(D)<w(D) \exp \left(\beta t_{1} J\right)<\exp \left\{-\beta t_{1}(|\operatorname{supp} D|-J) / 3 N_{b}\right\}
$$

Proof. We prove this lemma by using the method of the Peierls argument.

According to the definition, the probability of $D$ is

$$
P(D)=\frac{\sum^{1} w(D) w\left(D_{1}\right) \cdots w\left(D_{k}\right)}{\Xi(V)}
$$

where $w(D)=\prod_{j=1}^{k} w\left(D_{j}\right)$, and the summation in $\Sigma^{1}$ is taken over all nonordered compatible collections $D, D_{1}, \ldots, D_{k}$ of clusters containing $D$. The partition function is

$$
\Xi(V)=\sum w\left(D_{1}\right) \cdots w\left(D_{m}\right)
$$

where the summation is taken over all nonordered compatible collections of clusters.

Let us consider an arbitrary term $w(D) w\left(D_{1}\right) \cdots w\left(D_{m}\right)=Z_{j}^{l}$ from the numerator of (87).

According to Lemma 13, there exists a configuration $\varphi^{\prime}(x)$ such that $D \in B\left(\varphi^{\prime}(x)\right), D_{i} \in B(\varphi(x)), i=1, \ldots, m$.

Let us consider the configuration $\varphi_{l}^{\text {del, } 2}(x)$ corresponding to the configuration $\varphi^{l}(x)$ (see the proof of Lemma 12). Let

$$
\operatorname{supp} \bar{D}=\operatorname{supp} D \cap B\left(\varphi_{l}^{\text {del, } 2}(x)\right)
$$

and

$$
\bar{D}=\left(\varphi^{l}(\operatorname{supp} \bar{D}), \operatorname{supp} \bar{D}\right)
$$

In the denominator of (87) obviously there is the same term $Z_{j}^{l}$. Consider the following expression:

$$
\bar{Z}_{j}^{l}=w(\bar{D}) w\left(D_{1}\right) \cdots w\left(D_{m}\right)
$$

where $w(\bar{D})$ is defined as $(85)$. 
(a)

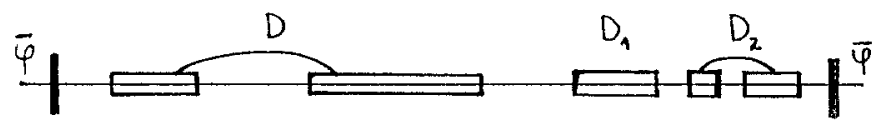

(b)

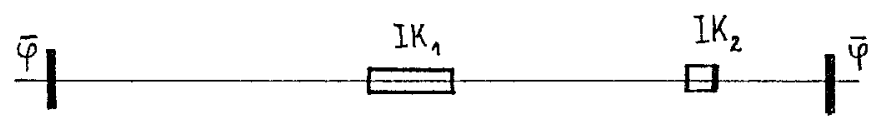

(c)

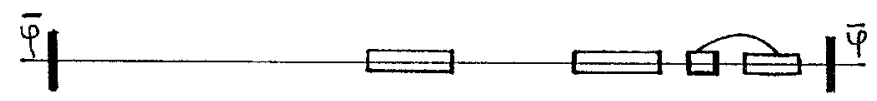

Fig. 2.

Figure $2 \mathrm{a}$ shows a collection of clusters corresponding to the term $Z_{j}^{l}=w(D) w\left(D_{1}\right) w\left(D_{2}\right)$, Fig. 2b shows a contours of the configuration $\varphi_{l}^{\text {del, } 2}(x)$, and Fig. $2 \mathrm{c}$ shows a collection of clusters corresponding to the term $\bar{Z}_{j}^{l}$.

Now consider the ratio

$Z_{j}^{l} / \bar{Z}_{j}^{l}=w(D) w\left(D_{1}\right) \cdots w\left(D_{m}\right) / w(\bar{D}) w\left(D_{1}\right) \cdots w\left(D_{m}\right)=w(D) / w(\bar{D})$

According to Lemmas 6-8 (note that $|\operatorname{supp} D|>\left|\operatorname{supp}^{1} D\right| 3 N_{b}$ ),

$$
\begin{aligned}
w(D) / w(\bar{D}) & <\exp \left\{-\beta t_{1}\left(|\operatorname{supp} D|-\left|B\left(\varphi_{I}^{\text {del, }, 2}(x)\right)\right|-N_{b}\left(n_{0}+1\right)\right) / 3 N_{b}\right\} \\
& <\exp \left\{-\beta t_{1}\left(|\operatorname{supp} D|-J_{1}-N_{b}\left(n_{0}+1\right)\right) / N_{b}\right\} \\
& =\exp \left\{-\beta t_{1}(|\operatorname{supp} D|-J) / N_{b}\right\}
\end{aligned}
$$

Finally,

$$
Z_{j}^{l} / \bar{Z}_{j}^{l}<\exp \left\{-\beta t_{1}(|\operatorname{supp} D|-J) / N_{b}\right\}
$$

Now we are ready to complete the proof. Indeed,

$$
\begin{aligned}
P(D) & =\frac{\sum^{1} w(D) w\left(D_{1}\right) \cdots w\left(D_{k}\right)}{\Xi(V)} \\
& =\frac{\sum^{1} Z_{j}^{l}}{\Xi(V)} \leqslant \frac{\sum^{1} Z_{j}^{l}}{\sum Z_{j}^{l}}<\exp \left\{-\beta t_{1}(|\operatorname{supp} D|-J)\right\}
\end{aligned}
$$

where the last inequality is fulfilled due to the inequality (90).

Lemma 15 is proved.

Lemma 16. Suppose that the value of the external field $\mu$ of the model (1) belongs to the interval $\left(\mu_{\kappa}^{-}, \mu_{\kappa}^{+}\right)$for some number $\kappa=q / p=1 / n_{0}$, 
and boundary conditions $\bar{\varphi}(x)=[\varphi(x), x \in(-\infty,-V-1] \cup[V+1, \infty)]$ are fixed.

Then for any large enough $\beta$ there exists $N_{b}=N_{b}(\beta)$, such that, for an arbitrary cluster $D$ such that supp $D \in[-V, V]$

$$
F(D)=\sum_{\mathbf{D}: D \subset \mathbf{D}} w(\mathbf{D})<\exp \left(-\beta t_{2}\left|\operatorname{supp}^{1} D\right|\right)
$$

where $t_{2}=t_{1} / 3$.

Proof. Let $D$ be an arbitrary cluster containing $K: K \in D$. We say that a contour $K^{\prime} \in D$ is a neighbor of the first order of a contour $K$ in a cluster $D$ and write $K^{\prime} \leftrightarrow K$ provided $K^{\prime}$ and $K$ are connected (see Definition 3). A contour $K^{\prime \prime}$ is called a neighbor of the $q$ th order for a contour $K$ provided $K \leftrightarrow K_{1} \leftrightarrow \cdots \leftrightarrow K_{q-1} \leftrightarrow K^{\prime \prime}$ and there are no such diagrams with fever arrows. Therefore, with a fixed contour $K \in D$, all the other contours of a given cluster $D$ are divided into nonintersecting classes indicated by the integers $1, \ldots, p$ of contours that are neighbors of the $q$ th order for the contour $K, q=1, \ldots, p(D, K)$. The number $p(D, K)$ is called the order of the cluster $D$ with respect to the contour $K$. The contours that are neighbors of $q$ th order of a fixed contour $K$ will be denoted by $K_{q}(K)$.

Besides the weight $w(K)$ [see (79)] we introduce the new weight

$$
\bar{w}_{c}(K)=w(K) \exp \left(\beta c\left|\operatorname{supp}^{1} K\right|\right)
$$

According to Lemma 5,

$$
\bar{w}_{c}(K)<\exp \left\{-\beta\left(t_{1}-c\right) \operatorname{supp}^{1} K\right\}
$$

First we prove the following inequality:

$$
\begin{aligned}
F^{1}(K) & =\sum_{D: D=\left(\left(K, K^{\prime}\right) ; G^{\prime}\right)} w(D) w_{c}\left(K^{\prime}\right) \prod_{(x, y) \in G^{\prime}} g(x, y) \\
& <\exp \left\{-\beta\left(t_{1}-c\right) \operatorname{supp}^{1} K\right\}
\end{aligned}
$$

It can be shown that

$$
F^{1}(K) \leqslant w(K) \prod^{1}
$$

where

$$
\begin{aligned}
& \prod^{1}=\prod_{x \in \operatorname{supp} K}\left\{1+\sum_{y: \operatorname{dist}(y, \operatorname{supp} K)>N_{b}}|g(x, y)|\left(1+\mathbf{Q}_{c}\right)\right\} \\
& \mathbf{Q}_{c}=\sum_{K: y \in \operatorname{supp} K} w_{c}(K)
\end{aligned}
$$


Now note that

$$
\mathbf{Q}_{c}<1
$$

if

$$
\beta\left(t_{1}-c\right) / 3 N_{b}>2 \ln 2+1
$$

In fact,

$$
\mathbf{Q}_{c} \leqslant \sum_{m=1}^{\infty} \sum_{K: y \in \operatorname{supp} K ;|\operatorname{supp} K|=m} \exp \left\{-\beta\left(t_{1}-c\right)\left(\left|\operatorname{supp}^{1} K\right|\right)\right\}
$$

According to Definition 2, $\left|\operatorname{supp}^{1} K\right| \geqslant|\operatorname{supp} K| / 3 N_{b}$, and

$$
\begin{aligned}
\mathbf{Q}_{c} & \leqslant \sum_{m=1}^{\infty} m 2^{m} \exp \left\{-\beta\left(t_{1}-c\right)\left(m / 3 N_{b}\right)\right\} \\
& \leqslant \sum_{m=1}^{\infty} \exp \left\{-\left(\beta\left(t_{1}-c\right) / 3 N_{b}-(\ln 2+1)\right) m\right\} \\
& <2 \exp \left\{-\left(\beta\left(t_{1}-c\right) / 3 N_{b}-(\ln 2+1)\right)\right\}<1
\end{aligned}
$$

Now we estimate the product $\Pi^{1}$.

By using (94), we have

$$
\begin{aligned}
\prod^{1} & \leqslant \prod_{x \in \operatorname{supp} K}\left\{1+\sum_{y: \operatorname{dist}(y, \operatorname{supp} K)>N_{b}} 2 g(x, y)\right\} \\
& \leqslant \exp \left\{\sum_{x \in \operatorname{supp} K} \ln \left(1+\sum_{y: \operatorname{dist}(y, \operatorname{supp} K)>N_{b}} 2|g(x, y)|\right)\right\} \\
& \leqslant \exp \left\{\sum_{x \in \operatorname{supp} K} y: \operatorname{dist}(y, \operatorname{supp} K)>N_{b}\right.
\end{aligned}
$$

Therefore,

$$
F^{1}(K) \leqslant w(K) \prod^{1} \leqslant w(D) \exp \left\{\sum_{x \in \operatorname{supp} K} \sum_{y: \operatorname{dist}(y, \operatorname{supp} K)>N_{b}} 2|g(x, y)|\right\}
$$

Note that

$$
|g(x, y)|=|\exp \{-\beta f(x, y)\}-1| \leqslant 2 \beta|f(x, y)|
$$

when

$$
\beta|f(x, y)|<1
$$


Finally.

$$
\begin{aligned}
F^{1}(K) & \leqslant w(K) \exp \left\{\sum_{x \in \operatorname{supp} K} \sum_{y: \operatorname{dist}(y, \operatorname{supp} D)>N_{b}} 4 \beta|f(x, y)|\right\} \\
& \leqslant w(K) \exp (4 \beta|G(D)|) \\
& \leqslant \exp \left(-\beta t_{1}\left|\operatorname{supp}^{1} K\right| / 3\right)
\end{aligned}
$$

The last inequality follows from Lemma 14 .

Therefore inequality (93) is fulfilled with $c=2 t / 3$ when the conditions (95) and (96) hold.

Let $D=\left[\left(K_{i}, i=1, \ldots, s\right) ; G^{\prime}\right]$. Now by induction on the order of a cluster and employing (93), we obtain the following inequality:

$$
\begin{aligned}
F(D) & =\sum_{\mathbf{D}: D \subset \mathbf{D}} w(\mathbf{D}) \\
& \leqslant \prod_{i=1}^{s} w_{c}\left(K_{i}\right) \prod_{(x, y) \in G^{\prime}} g(x, y) \\
& =\exp \left(\beta c\left|\operatorname{supp}^{1} D\right|\right) \prod_{i=1}^{s} w\left(K_{i}\right) \prod_{(x, y) \in G^{\prime}} g(x, y) \\
& \leqslant \exp \left(\beta c\left|\operatorname{supp}^{1} D\right|\right) \exp \left(-\beta t_{1}\left|\operatorname{supp}^{1} D\right|\right)
\end{aligned}
$$

The last inequality follows from Lemma 14.

Finally,

$$
F(D) \leqslant \exp \left(-t_{1} \beta\left|\operatorname{supp}^{1} D\right| / 6\right)
$$

Lemma 16 is proved.

Lemma 17. Suppose that the value of the external field $\mu$ of the model (1) belongs to the interval $\left(\mu_{\kappa}^{-}, \mu_{\kappa}^{+}\right)$for some number $\kappa=q / p=1 / n_{0}$, and boundary conditions $\bar{\varphi}(x)=[\varphi(x), x \in(-\infty,-V-1] \cup[V+1, \infty)]$ are fixed.

Then for any $\beta$ large enough there exists $N_{b}=N_{b}(\beta)$ such that for an arbitrary subset $Y$ of the segment $[-V, V]$ such that $|Y|>4 J$ ( $J$ is a constant defined in Lemma 15), we have the probability

$$
\begin{aligned}
\mathbf{P}\left(\mathbf{A}_{Y}\right)= & \mathbf{P}(Y \text { does not contain any segment }[a, b], \\
& \text { such that } \varphi([a, b]) \text { is a regular phase } \\
< & \exp \left\{-\beta t_{3}(|Y| / 4-J)\right\}
\end{aligned}
$$

where $t_{3}=t_{1} / 6 N_{b}$. 
Proof. According to the definitions,

$$
\mathbf{P}\left(\mathbf{A}_{Y}\right)=\sum_{\varphi(x) \in \Phi(V)} \mathbf{P}(\varphi(x))=\sum_{\text {D: } Y \subset \operatorname{supp} D \text { and } D \in \mathbf{D}} \mathbf{P}(\mathbf{D})
$$

Suppose that $Y=\bigcup_{i=1}^{s} Y_{i}$, where $Y_{i}, i=1, \ldots, s$, are segments such that $\operatorname{dist}\left(Y_{k}, Y_{l}\right)>N_{b}$. Then

$$
\mathbf{P}\left(\mathbf{A}_{Y}\right) \leqslant \sum_{m_{1}, \ldots, m_{s}=0}^{\infty} \sum^{1} \mathbf{P}(\mathbf{D})=\sum_{m_{1}, \ldots, m_{s}=0}^{\infty} \mathbf{S}\left(m_{1}, \ldots, m_{s}\right)
$$

where the sum $\sum^{1}$ is taken over all the clusters $\mathbf{D}: K_{i} \subset \mathbf{D}, i=1, \ldots, s$; $Y_{i} \subset K_{i} ;\left|\operatorname{supp} K_{i}\right|=\left|Y_{i}\right|+m_{i}$.

According to Lemma 15,

$$
\mathbf{P}(D)<w(D) \exp \left(\beta t_{1} J\right)<\exp \left\{-\beta t_{1}(|\operatorname{supp} D|-J) / 3 N_{b}\right\}
$$

Therefore,

$$
\mathbf{S}\left(m_{1}, \ldots, m_{s}\right)<\exp \left(\beta t_{1} J / 3 N_{b}\right) \sum^{1} w(D)
$$

Now note that $\left|\operatorname{supp}^{1} D\right|>|\operatorname{supp} D| / 3 N_{b}$, there are at most $2^{\mid Y_{i i}+m_{i}}$ contours with $\left|\operatorname{supp} K_{i}\right|=\left|Y_{i}\right|+m_{i}$, and the distance between the left end of the contour $K_{i}$ and the set $Y$ is not greater than $m_{i}$. By using inequality (91) from Lemma 16, we get

$$
\begin{aligned}
\mathbf{S}\left(m_{1}, \ldots, m_{s}\right) & <\exp \left(\beta t_{1} J / 3 N_{b}\right) \prod_{i=1}^{s} 2^{m_{i}} m_{i} \exp \left\{-\beta t_{2}\left(\left|Y_{i}\right|+m_{i}\right) / 3 N_{b}\right\} \\
& =\exp \left(\beta t_{1} J / 3 N_{b}\right) \exp \left\{-\beta t_{2}(|Y|+m) / 3 N_{b}+m(\ln 2+1)\right\}
\end{aligned}
$$

Finally,

$$
\begin{aligned}
\mathbf{P}\left(\mathbf{A}_{Y}\right) & \leqslant \sum_{m_{1}, \ldots, m_{s}}^{\infty} \mathbf{S}\left(m_{1}, \ldots, m_{s}\right) \\
& \leqslant \exp \left(\beta t_{1} J / 3 N_{b}\right) \prod_{i=1}^{s} \sum_{m_{i}=1}^{\infty} 2^{m_{i}} m_{i} \exp \left\{-\beta t_{2}\left(\left|Y_{i}\right|+m_{i}\right) / 3 N_{b}\right\} \\
& \leqslant \exp \left(\beta t_{1} J / 3 N_{b}\right) \prod_{i=1}^{s} \exp \left(-\beta t_{2}\left|Y_{i}\right| / 3 N_{b}+3\right) \\
& \leqslant \exp \left(\beta t_{1} J / 3 N_{b}\right) \exp \left(-\beta t_{2}|Y| / 3 N_{b}+3|Y|\right) \\
& \leqslant \exp \left\{-\beta t_{1}(|Y| / 4-J) / 3 N_{b}\right\} \exp \left(-\beta t_{2}|Y| / 6 N_{b}+3|Y|\right)
\end{aligned}
$$


if

$$
\beta t_{2} / 3 N_{b}>\ln 2+1
$$

Finally,

$$
\mathbf{P}\left(\mathbf{A}_{Y}\right)<\exp \left\{-\beta t_{1}(|Y| / 4-J) / 3 N_{b}\right\}
$$

if

$$
\beta t_{2}>18 N_{b}
$$

Lemma 17 is proved.

Lemma 17 allows us to describe the set of all typical configurations of the model (1) at low temperatures.

Now we have completed the proofs of all preliminary lemmas and hence pass to the proof of the uniqueness of the Gibbs states.

\section{UNIQUENESS OF THE GIBBS STATES. \\ THE DENSITY $\mathrm{K}$ IS $1 / \boldsymbol{n}_{0}$}

In this section we prove the following result.

Theorem 5. Suppose that the value of the external field $\mu$ of the model (1) belongs to the interval $\left(\mu_{\kappa}^{-}, \mu_{\kappa}^{+}\right)$for some number $\kappa=q / p=1 / n_{0}$.

Then the model (1) has a unique Gibbs state at all sufficiently small values of temperature $\left[\beta^{-1}<\operatorname{const}(\mu, U(x))\right]$.

First we prove the following key lemma.

Let $\mathbf{P}^{1}$ and $\mathbf{P}^{2}$ be two Gibbs states of the model (1) corresponding to the boundary conditions $\varphi^{1}(x)$ and $\varphi^{2}(x)$, respectively.

Lemma 18. Suppose that the value of the external field $\mu$ of the model (1) belongs to the interval $\left(\mu_{\kappa}^{-}, \mu_{\kappa}^{+}\right)$for some number $\kappa=q / p=1 / n_{0}$.

Then the measures $\mathbf{P}^{1}$ and $\mathbf{P}^{2}$ are absolutely continuous with respect to each other.

Proof. Let $I=[a, b]$ be an arbitrary segment and $\varphi^{\prime}(I)$ be an arbitrary configuration. In order to prove the lemma, we show that there exist two positive constants $s$ and $S$ not depending on $I$ and $\varphi^{\prime}(I)$ such that

$$
s \leqslant \mathbf{P}^{1}\left(\varphi^{\prime}(I)\right): \mathbf{P}^{2}\left(\varphi^{\prime}(I)\right) \leqslant S
$$

Let $\mathbf{P}_{N}^{1}$ and $\mathbf{P}_{N}^{2}$ be Gibbs measures corresponding to the boundary conditions $\varphi^{1}(x)$ and $\varphi^{2}(x), x \in \mathbf{Z}^{1}-I_{N}$, respectively, where $I_{N}=(-\infty$, $-N-1] \cup[N+1,+\infty)$. 
By assumption,

$$
\lim _{N \rightarrow \infty} \mathbf{P}_{N}^{1}=\mathbf{P}^{1} \quad \text { and } \quad \lim _{N \rightarrow \infty} \mathbf{P}_{N}^{2}=\mathbf{P}^{2}
$$

where we consider a weak convergence of probability measures.

In order to establish the inequality (99), it will be shown that for each fixed interval $I, I \subset[-M, M]$, there exists number $N_{0}(M)$, which depends on $M$ only, such that

$$
s \leqslant \mathbf{P}_{N}^{1}\left(\varphi^{\prime}(I)\right): \mathbf{P}_{N}^{2}\left(\varphi^{\prime}(I)\right) \leqslant S
$$

if $N>N_{0}$.

Consider

$$
\begin{aligned}
\mathbf{P}_{N}^{1}\left(\varphi^{\prime}(I)\right) & =\frac{\sum_{\varphi\left(I_{N}\right): \varphi(I)=\varphi^{\prime}(I)} \exp \left\{-\beta H\left(\varphi\left(I_{N}\right) \mid \varphi^{1}(x)\right)\right\}}{\sum_{\varphi\left(I_{N}\right)} \exp \left\{-\beta H\left(\varphi\left(I_{N}\right) \mid \varphi^{1}(x)\right)\right\}} \\
& =\frac{\Xi\left(I_{N}-I \mid \varphi^{1}(x), \varphi^{\prime}(I)\right)}{\sum_{\varphi^{\prime \prime}(I)} \Xi\left(I_{N}-I \mid \varphi^{1}(x), \varphi^{\prime \prime}(I)\right)}
\end{aligned}
$$

where $\Xi\left(I_{N}-I \mid \varphi^{1}(x), \varphi^{\prime \prime}(I)\right)$ denotes the partition corresponding to the boundary conditions $\varphi^{1}(x), x \in \mathbf{Z}^{1}-I_{N}$, and $\varphi^{\prime}(I), x \in I$.

We can express $\mathbf{P}_{N}^{2}\left(\varphi^{\prime}(I)\right)$ in just the same way.

In order to prove the inequality (100), it is enough to show that

$$
\left|G\left(\varphi(I), N, \varphi^{i}(x)\right)\right|=\sum_{(x, y) \in \operatorname{Int}\left(I, \mathbf{Z}^{1}-I_{N}\right)}|f(x, y)|<1, \quad i=1,2
$$

and

$$
\frac{1}{S} \leqslant \frac{\Xi\left(I_{N}-I \mid \varphi^{1}(x), \varphi^{\prime \prime}(I)\right)}{\Xi\left(I_{N}-I \mid \varphi^{1}(x), \varphi^{\prime}(I)\right)}: \frac{\Xi\left(I_{N}-I \mid \varphi^{2}(x), \varphi^{\prime \prime}(I)\right)}{\Xi\left(I_{N}-I \mid \varphi^{2}(x), \varphi^{\prime}(I)\right)} \leqslant \frac{1}{S}
$$

for arbitrary $\varphi^{\prime \prime}(I)$.

If the inequalities (101) and (102) hold, then

$$
\begin{aligned}
\mathbf{P}_{N}^{1}\left(\varphi^{\prime}(I)\right): \mathbf{P}_{N}^{2}\left(\varphi^{\prime}(I)\right) \\
=1:\left(\frac{\sum_{\varphi^{\prime \prime}(I)} \Xi\left(I_{N}-I \mid \varphi^{1}(x), \varphi^{\prime \prime}(I)\right)}{\Xi\left(I_{N}-I \mid \varphi^{1}(x), \varphi^{\prime}(I)\right)}: \frac{\sum_{\varphi^{\prime \prime}(I)} \Xi\left(I_{N}-I \mid \varphi^{2}(x), \varphi^{\prime \prime}(I)\right)}{\Xi\left(I_{N}-I \mid \varphi^{2}(x), \varphi^{\prime}(I)\right)}\right) \\
=1: \frac{\left(\sum_{\varphi^{\prime \prime}(I)} \Xi\left(I_{N}-I \mid \varphi^{1}(x), \varphi^{\prime \prime}(I)\right)\right) \Xi\left(I_{N}-I \mid \varphi^{2}(x), \varphi^{\prime}(I)\right)}{\left(\sum_{\varphi^{\prime \prime}(I)} \Xi\left(I_{N}-I \mid \varphi^{2}(x), \varphi^{\prime \prime}(I)\right)\right) \Xi\left(I_{N}-I \mid \varphi^{1}(x), \varphi^{\prime}(I)\right)}
\end{aligned}
$$

Therefore,

$$
1: 1 / s \leqslant \mathbf{P}_{N}^{1}\left(\varphi^{\prime}(I)\right): \mathbf{P}_{N}^{2}\left(\varphi^{\prime}(I)\right) \leqslant 1: 1 / S
$$


since the quotient of $\sum_{i=1}^{n} a_{i}: \sum_{i=1}^{n} b_{i}$ lies between $\min \left(a_{i} / b_{i}\right)$ and $\max \left(a_{i} / b_{i}\right)$.

The inequality (101) easily follows from Lemma 4 . Indeed, according to Lemma 4 ,

$$
\left|G\left(\varphi(I), N, \varphi^{i}(x)\right)\right| \leqslant C_{1}|N-M|^{-\alpha} M^{1-\alpha}
$$

and hence for each fixed $M$ there exists $N_{0}$ such that if $N>N_{0}$, then $\left|G\left(\varphi(I K), N, \varphi^{i}(x)\right)\right|<1$.

Thus, for completing the proof of Lemma 18, we have to prove the following inequality [which is just the transformed inequality (102)]:

$$
\frac{1}{S} \leqslant \frac{\Xi\left(I_{N}-I \mid \varphi^{1}(x), \varphi^{\prime \prime}(I)\right) \Xi\left(I_{N}-I \mid \varphi^{2}(x), \varphi^{\prime}(I)\right)}{\Xi\left(I_{N}-I \mid \varphi^{2}(x), \varphi^{\prime \prime}(I)\right) \Xi\left(I_{N}-I \mid \varphi^{1}(x), \varphi^{\prime}(I)\right)}=\frac{\Xi^{1, "} \Xi^{2, \prime}}{\Xi^{2, "} \Xi^{1, \prime}} \leqslant \frac{1}{s}
$$

Consider one of the four partition functions from the previous inequality, say $\Xi^{1, \prime}$. According to Lemma 13,

$$
\Xi^{1, \prime}=\sum^{1, \prime} w\left(D_{1}^{1, \prime}\right) \cdots w\left(D_{m}^{1, \prime}\right)
$$

where the summation in $\Sigma^{1, \prime}$ is taken over all nonordered compatible collections of clusters $\left[D_{1}^{1, \prime} \cdots D_{m}^{1, \prime}\right], \bigcup_{i=1}^{m} \operatorname{supp} D_{i}^{1, \prime^{\prime}} \subset I_{N}-I$ corresponding to the boundary conditions $\varphi^{1}(x), x \in \mathbf{Z}^{1}-I_{N}$, and $\varphi^{\prime}(x), x \in I$.

A cluster $D=\left[\left(K_{i}, i=1, \ldots, r\right) ; G^{\prime}\right]$ of the above sum is said to be long if the diameter of the set $\left(\bigcup_{i=1}^{m} \operatorname{supp} D_{i}\right) \cup G^{\prime}$ is greater than $\left(N-M-N_{b}\right) / 2\left(N_{b}\right.$ is defined in Definition 2) and the set

$$
\left.\left(\left(\bigcup_{i=1}^{m} \operatorname{supp} D_{i}\right) \cup G^{\prime}\right) \cap\left(\left(\mathbf{Z}^{1}-I_{N}\right) \cup I\right)\right)
$$

is not empty.

Lemma 19. Let $h_{1}$ and $h_{2}$ be positive constants such that $0<h_{1}<$ $1<h_{2}$. Then for each fixed interval $I, I \subset[-M, M]$, there exists a number $N_{0}(M)$, which depends on $M$ only, such that if $N>N_{0}$,

$$
\begin{gathered}
\Xi^{1, \prime}=h^{1, \prime} \sum w\left(D_{1}^{1, \prime}\right) \cdots w\left(D_{m}^{1, \prime}\right)=h^{1, \prime} \Xi_{0}^{1, \prime} \\
h_{1} \leqslant h^{1, \prime} \leqslant h_{2}
\end{gathered}
$$

where the summation is taken over all nonlong, nonordered compatible collections of clusters $\left[D_{1}^{1, \prime} \cdots D_{m}^{1, \prime}\right], \bigcup_{i=1}^{m} \operatorname{supp} D_{i}^{1, \prime} \subset I_{N}-I$, corresponding to the boundary conditions $\varphi^{1}(x), x \in \mathbf{Z}^{1}-I_{N}$, and $\varphi^{\prime}(x), x \in I$. 
Proof. Let $X\left(I, \varphi^{\prime}(x), N, M\right)=\sum w(D)$, where the summation is taken over all long clusters $D$ [hence the length of each cluster is greater than $\left.\left(N-M-N_{b}\right) / 2\right]$. We can repeat the arguments used in the proof of Lemma 4 and prove the following inequality:

$$
\ln X<|I|^{1-\alpha}\left(\left(N-M-N_{b}\right) / 2\right)^{-\alpha}
$$

and by choosing sufficiently large $N$ to prove Lemma 19 .

But we prefer another way. From the proof of Lemma 16 [see (91)] it is known that for each fixed $M$

$$
\sum_{N=M}^{\infty} \ln X\left(I, \varphi^{\prime}(x), N, M\right)<\mathrm{const}
$$

Therefore, $\lim _{N-M-N_{b} \rightarrow \infty} \ln X\left(I, \varphi^{\prime}(x), N, M\right)=0$ and hence for each $M$ and $h_{1}, h_{2}\left(0<h_{1}<1<h_{2}\right)$ there exists $N_{0}$ such that

$$
h_{1}<X\left(I, \varphi^{\prime}(x), N, M\right)<h_{2}
$$

if $N>N_{0}$.

Now we can complete the proof. In fact,

$$
\Xi^{1, \prime}=\left(\Xi^{1, \prime} / \Xi_{0}^{1, \prime}=h^{1, '} \Xi_{0}^{1, \prime}\right.
$$

Consider $h^{1,{ }^{\prime}}=\Xi^{1, \prime} / \Xi_{0}^{1,{ }^{\prime}}$.

Note that if $\left[D_{1}^{1,} \ldots D_{m}^{1, \prime}\right]$ is some term of the sum in the denominator, then the sum of all terms from the numerator including $\left[D_{1} \cdots D_{m}\right]$ is less than $w\left(D_{1}\right) \cdots w\left(D_{m}\right) X\left(I, \varphi^{\prime}(x), N, M\right)$. Therefore,

$$
h_{1} \leqslant h^{1, \prime} \leqslant h_{2}
$$

Lemma 19 is proved.

According to Lemma 19,

$$
\Xi^{1, \prime}=h^{1, \prime} \Xi_{0}^{1, \prime}, \quad \Xi^{1, \prime \prime}=h^{1, \prime \prime} \Xi_{0}^{1, \prime \prime}, \quad \Xi^{2, \prime}=h^{2, \prime} \Xi_{0}^{2, \prime}, \quad \Xi^{2, \prime \prime}=h^{2, " \prime} \Xi_{0}^{2, \prime \prime}
$$

where

$$
h_{1} \leqslant h^{1, \prime} \leqslant h_{2}, \quad h_{1} \leqslant h^{1, \prime} \leqslant h_{2}, \quad h_{1} \leqslant h^{2, \prime} \leqslant h_{2}, \quad h_{1} \leqslant h^{2, "} \leqslant h_{2}
$$

Therefore, the validity of inequality (103) with

$$
1 / s=Q_{2} h_{2}^{2} / h_{1}^{2}, \quad 1 / S=Q_{1} h_{1}^{2} / h_{2}^{2}
$$

directly follows from the following result. 
Lemma 20. For each fixed interval $I, I \subset[-M, M]$ there exists a number $N_{0}(M)$ such that if $N>N_{0}(M)$

$$
Q_{1} \leqslant \frac{\Xi_{0}^{1, "} \Xi_{0}^{2, \prime}}{\Xi_{0}^{2, "} \Xi_{0}^{1, \prime}} \leqslant Q_{2}
$$

where the two positive constants $Q_{1}$ and $Q_{2}$ do not depend on $\varphi^{\prime}(I), \varphi^{\prime \prime}(I)$, $\varphi^{1}(x)$, and $\varphi^{2}(x)$.

Proof. Consider $\Xi_{0}^{1, "} \Xi_{0}^{2, \prime}$. By definition and according to Lemma 13 ,

$$
\Xi^{1, "} \Xi^{2, \prime}=\sum^{1, \prime \prime} \sum^{2, \prime} w\left(D_{1}^{1, \prime \prime}\right) \cdots w\left(D_{m}^{1, \prime \prime}\right) w\left(D_{1}^{2, \prime}\right) \cdots w\left(D_{k}^{2, \prime}\right)
$$

where the summation in $\Sigma^{1, "}$ is taken over all nonlong, nonordered compatible collections of clusters $\left[D_{1}^{1, "} \cdots D_{m}^{1, \prime \prime}\right], \bigcup_{i=1}^{m} \operatorname{supp} D_{i}^{1, " \prime} \subset I_{N}-I$, corresponding to the boundary conditions $\varphi^{1}(x), x \in \mathbf{Z}^{1}-I_{N}$, and $\varphi^{\prime \prime}(x)$, $x \in I$, and the summation in $\Sigma^{2, \prime}$ is taken over all nonlong, nonordered compatible collections of clusters $\left[D_{1}^{2, \prime} \cdots D_{k}^{2, \prime}\right], \bigcup_{i=1}^{k} \operatorname{supp} D_{i}^{2, \prime} \subset I_{N}-I$, corresponding to the boundary conditions $\varphi^{2}(x), x \in \mathbf{Z}^{1}-I_{N}$, and $\varphi^{\prime}(x)$, $x \in I$.

In just the same way we get

$$
\Xi^{2, \prime \prime} \Xi^{1, \prime}=\sum^{2, \prime \prime} \sum^{1, \prime} w\left(D_{1}^{2, \prime \prime}\right) \cdots w\left(D_{l}^{2, \prime \prime}\right) w\left(D_{1}^{1, \prime}\right) \cdots w\left(D_{n}^{1, \prime}\right)
$$

The sketch of the proof is the following. Let $\Xi^{1, "} \Xi^{2, \prime}=\Sigma^{3} w\left(D_{1}\right) \cdots w\left(D_{k}\right)$ and $\Xi^{2, "} \Xi^{1, \prime}=\Sigma^{4} w\left(D_{1}\right) \cdots w\left(D_{l}\right)$, where $\sum^{3}=\sum^{1, \prime \prime} \Sigma^{2, \prime}$ and $\Sigma^{4}=\sum^{2, \prime \prime} \sum^{1, \prime}$.

We define a sub-sum $\Sigma^{5} \subset \Sigma^{3}$ and a sub-sum $\Sigma^{6} \subset \Sigma^{4}$ and put one-toone correspondence between $\Sigma^{3}-\Sigma^{5}$ and $\Sigma^{4}-\Sigma^{6}$ and prove that $\Sigma^{5}$ and $\sum^{6}$ are negligible.

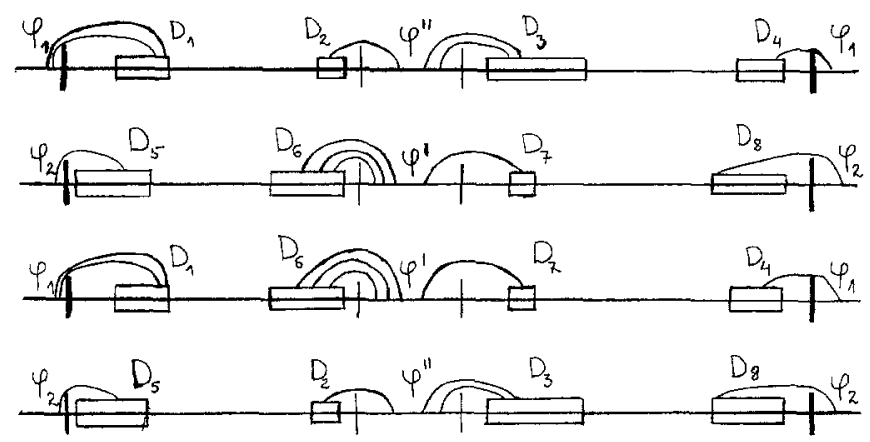

Fig. 3. 
A cluster $D=\left[\left(K_{i}, i=1, \ldots, r\right) ; G^{\prime}\right]$ is said to be basic if the set

$$
\left.\left(\left(\bigcup_{i=1}^{m} \operatorname{supp} D_{i}\right) \cup G^{\prime}\right) \cap\left(\left(\mathbf{Z}^{1}-I_{N}\right) \cup I\right)\right)
$$

is not empty. In Fig. 3 all clusters are basic.

Let

$$
U=w\left(D_{1}\right) \cdots w\left(D_{m+k}\right)=w\left(D_{1}^{1, \prime \prime}\right) \cdots w\left(D_{m}^{1, \prime \prime}\right) w\left(D_{1}^{2, \prime}\right) \cdots w\left(D_{k}^{2, \prime}\right)
$$

be some term of $\Sigma^{3}$. Consider $W=\bigcup_{i=1}^{m} D_{i}^{1, \prime \prime} \bigcup_{i=1}^{k} D_{i}^{2, \prime}$ [note that $W$ is the set of all clusters of the term $\left.w\left(D_{1}\right) \cdots w\left(D_{k}\right)\right]$ and four subsets of $W$ :

$$
\begin{aligned}
& W^{\prime}=\left[D^{\prime}=\left[\left(K_{i}, i=1, \ldots, r\right) ; G^{\prime}\right] \in \Xi^{2, \prime}:\right. \\
& \left.\left(\left(\bigcup_{i=1}^{k} \operatorname{supp} D_{i}\right) \cup G^{\prime}\right) \cap I \text { is not empty }\right] \\
& W^{\prime \prime}=\left[D^{\prime \prime}=\left[\left(K_{i}, i=1, \ldots, r\right) ; G^{\prime}\right] \in \Xi^{1, "}\right. \text { : } \\
& \left.\left(\left(\bigcup_{i=1}^{m} \operatorname{supp} D_{i}\right) \cup G^{\prime}\right) \cap I \text { is not empty }\right] \\
& W^{1}=\left[D^{1}=\left[\left(K_{i}, i=1, \ldots, r\right) ; G^{\prime}\right] \in \Xi^{1, "}\right. \text { : } \\
& \left.\left(\left(\bigcup_{i=1}^{m} \operatorname{supp} D_{i}\right) \cup G^{\prime}\right) \cap\left(\mathbf{Z}^{1}-I_{N}\right) \text { is not empty }\right] \\
& W^{2}=\left[D^{2}=\left[\left(K_{i}, i=1, \ldots, r\right) ; G^{\prime}\right] \in \Xi^{2, \prime}:\right. \\
& \left.\left(\left(\bigcup_{i=1} \operatorname{supp} D_{i}\right) \cup G^{\prime}\right) \cap\left(\mathbf{Z}^{1}-I_{N}\right) \text { is not empty }\right]
\end{aligned}
$$

Note that the subsets $W^{\prime}, W^{\prime \prime}, W^{1}$, and $W^{2}$ contain only basic clusters and their union contains all basic clusters of the term $U$.

Let

$$
w\left(D_{1}\right) \cdots w\left(D_{l+n}\right)=\Lambda w\left(D_{1}^{1, \prime}\right) \cdots w\left(D_{l}^{1, \prime}\right) w\left(D_{1}^{2, \prime \prime}\right) \cdots w\left(D_{n}^{2, \prime \prime}\right)
$$

be some term of $\sum^{4}$. Consider $W=\bigcup_{i=1}^{\prime} D_{1}^{i, \prime} \bigcup_{i=1}^{n} D_{1}^{i \prime \prime}$ [note that $W$ is the set of all clusters of the term $\left.w\left(D_{1}\right) \cdots w\left(D_{l+n}\right)\right]$. In just the same way we can define four subsets of $W$. 
Consider a term $U=w\left(D_{1}\right) \cdots w\left(D_{k}\right) \in \sum^{3}$ containing only basic clusters. By definition, $\bigcup_{i=1}^{k} D_{i}$ can bé represented as $\bigcup_{i=1}^{k} D_{i}=$ $\left(\bigcup_{i=1}^{m} D_{i}\right) \cup\left(\bigcup_{i=m+1}^{k} D_{j}\right)$, where the clusters $\bigcup_{i=1}^{m} D_{i}=W^{1} \cup W^{\prime}$ and $\bigcup_{i=m+1}^{k} D_{j}=W^{2} \cup W^{\prime \prime}$.

From the definition of nonlong clusters and $W^{\prime}, W^{\prime \prime}, W^{1}$, and $W^{2}$ it easily follows that there exists the same term $U^{\prime}=w\left(D_{1}\right) \cdots w\left(D_{k}\right) \in \Sigma^{4}$ such that $\bigcup_{i=1}^{k} D_{i}=\left(\bigcup_{i=1}^{m} D_{i}\right) \cup\left(\bigcup_{i=m+1}^{k} D_{j}\right)$, where the clusters $\bigcup_{i=1}^{m} D_{i}=$ $W^{1} \cup W^{\prime}$ and $\bigcup_{i=m+1}^{k} D_{j}=W^{2} \cup W^{\prime \prime}$.

Figure 3 shows four collections of clusters, $C O L_{1}=\left[D_{1}^{1, \prime \prime}, D_{2}^{1, \prime \prime}\right.$, $\left.D_{3}^{1, \prime \prime}, D_{4}^{1, \prime \prime}\right], C O L_{2}=\left[D_{5}^{2, \prime}, D_{6}^{2, \prime}, D_{7}^{2,}, D_{8}^{2, \prime}\right], C O L_{3}=\left[D_{1}^{1, \prime}, D_{6}^{1, \prime}, D_{7}^{1, \prime}, D_{4}^{1, \prime}\right]$, $\mathrm{COL}_{4}\left[D_{5}^{2, \prime \prime}, D_{2}^{2, \prime \prime}, D_{3}^{2, \prime \prime}, D_{8}^{2, \prime \prime}\right]$. Two coincident terms $U=U^{\prime}=\prod_{i=1}^{8} w\left(D_{i}\right)$ belonging to the sums $\sum^{3}$ and $\Sigma^{4}$ are constructed the by union of the collections $C O L_{1}, C O L_{2}$ and $C O L_{3}, C O L_{4}$, respectively.

We see that we easily can put a one-to-one correspondence between terms $U \in \Sigma^{3}$ and $U^{\prime} \in \Sigma^{4}$ containing only basic clusters.

Consider the term

$$
U=w\left(D_{1}\right) \cdots w\left(D_{k}\right) w\left(D_{k+1}\right) \cdots w\left(D_{n}\right) \in \Sigma^{3}
$$

containing basic clusters $D_{1} \cdots D_{k}$ and not basic clusters $D_{k+1} \cdots D_{n}$.

Now we have two cases:

1. There exists a term

$$
U^{\prime}=w\left(D_{1}\right) \cdots w\left(D_{k}\right) w\left(D_{k+1}\right) \cdots w\left(D_{n}\right) \in \sum^{4}
$$

coinciding with the term $U \in \Sigma^{3}$.

2. There is no term

$$
U^{\prime}=w\left(D_{1}\right) \cdots w\left(D_{k}\right) w\left(D_{k+1}\right) \cdots w\left(D_{n}\right) \in \sum^{4}
$$

coinciding with the term $U \in \Sigma^{3}$.

Let $\Sigma^{5}$ be te set of all terms $U \in \Sigma^{3}$ belonging to the second case. By the same way we define the set $\Sigma^{6} \subset \sum^{4}$.

It can be easily shown that in the second case the union of supports of all clusters contains at least a support of one long cluster (moreover, the diameter of this cluster is greater than $N-M$ ).

Now note that

$$
Q_{1} \leqslant \frac{\Sigma^{3}}{\sum^{4}}: \frac{\sum^{3}-\Sigma^{5}}{\sum^{4}-\Sigma^{6}} \leqslant Q_{2}
$$


Proof of the last inequality almost coincides with the proof of Lemma 19 and is omitted (the only difference is the existence of overlapping clusters in $\Sigma^{5}$ and $\Sigma^{6}$ ).

Lemma 20, and hence Lemma 18, are proved.

Let $\mathbf{P}^{1}$ and $\mathbf{P}^{2}$ be two different extreme Gibbs states of the model (1) corresponding to the boundary conditions $\varphi^{1}(x)$ and $\varphi^{2}(x)$, respectively.

Theorem 6. ${ }^{(17)} \mathbf{P}^{1}$ and $\mathbf{P}^{2}$ are singular or coincide.

Proof of Theorem 5. Let $\mathbf{P}^{1}$ and $\mathbf{P}^{2}$ be two different extreme Gibbs states of the model (1) corresponding to the boundary conditions $\varphi^{1}(x)$ and $\varphi^{2}(x)$, respectively. According to Lemma 18, $\mathbf{P}^{1}$ and $\mathbf{P}^{2}$ are not singular. Therefore, according to Theorem $6, \mathbf{P}^{1}$ and $\mathbf{P}^{2}$ coincide, which contradicts the assumption. Theorem 5 is proved.

\section{GROUND AND GIBBS STATES. THE DENSITY IS RATIONAL}

In this section we generalize the obtained results for all rational values of the density.

Suppose that the value of the external field $\mu$ of the model (1) belongs to the interval $\left(\mu_{\kappa}^{-}, \mu_{\kappa}^{+}\right)$for some number $\kappa=q / p=\left[n_{0}, n_{1}, \ldots, n_{s}\right]$.

The notions of the contour and interface contour are defined in Definitions 2 and 3. Now we show the validity of the important Lemma 1.

Proof of Lemma 1 for an arbitrary rational value of the density of the ground state.

1. Suppose that

$$
\sum_{x \in \operatorname{supp} K}\left(\varphi^{\prime}(x)-\varphi_{n_{0}, \ldots, n_{s}}(x)\right)=0
$$

This implies that the perturbation $\varphi(x)$ is obtained by shifting of some particles only.

By making use of Theorem 2 it can be easily shown that in this case the inequality

$$
H\left(\varphi^{\prime}(x)\right)-H\left(\varphi_{n_{0}}(x)\right) \geqslant t|\operatorname{supp} P B|
$$

is fulfilled with

$$
\begin{aligned}
& t=(U(p-1)+U(p+1)-2 U(p)) \theta \\
& \theta=\left(L-d_{0} p\right): 2 L\left(d_{0}+1\right) p
\end{aligned}
$$


2. Suppose that

$$
\left.\sum_{x \in \operatorname{supp} K} \varphi^{\prime}(x)-\varphi_{n_{0}, \ldots, n_{s}}(x)\right) \neq 0
$$

Let the density of the configuration $\varphi^{\prime}(x), x \in \operatorname{supp} K$, be $\kappa$ and the reciprocal of the density be $\eta$. Let $\eta=\left[\eta_{1}, \ldots, \eta_{s}\right]$, where $\eta_{s}$ is not necessarily integer. By assumption there exists an index $k, 1 \leqslant k \leqslant s$, such that $n_{i}=\eta_{i}$ for $i=0, \ldots, k-1$ and $n_{k} \neq \eta_{k}$. In this case we divide the proof into six cases.

Case $2 a . \quad n_{k}+1 \leqslant \eta_{k}$.

Case 2b. $n_{k}+1-\varepsilon<\eta_{k}<n_{k}+1$.

Case $2 c . \quad n_{k}<\eta_{k} \leqslant n_{k}+1-\varepsilon$.

Case $2 d$. $\eta_{k} \leqslant n_{k}-1$.

Case 2e. $n_{k}-1<\eta_{k}<n_{k}-1+\delta$.

Case 2f. $n_{k}-1+\delta \leqslant \eta_{k}<n_{k}$.

The last part of the proof is analogous to the proof of Lemma 1 for the special case of $\kappa=q / p=1 / n_{0}$ and will be omitted.

Now we can generalize all obtained results for an arbitrary rational value of the density. Thus, Theorem 4 holds for all rational densities; Theorem 3 is proved.

\section{CONCLUSIONS}

Thus we have proved that model (1) has a unique Gibbs state.

Theorem 7. ${ }^{(2)}$ Every Gibbs state of the model (1), $\mathbf{P}$, is translation invariant.

Note that our proof of Theorem 3 imposes on the value of the inverse temperature a very inconvenient condition, $\beta>\beta^{\text {cr }}[$ see (98)], where

$$
\lim _{p \rightarrow \infty} \beta^{\mathrm{cr}}=\infty
$$

where $p$ is the period of the special ground states.

This condition is unnatural. In fact, consider two values of the external field, say $\mu_{1}$ and $\mu_{2}$, with corresponding reciprocal densities of the special ground states $\kappa_{1}=n_{0}$ and $\kappa_{2}=n_{0}+1 / n_{1}$ and critical temperatures $\beta_{1}^{\text {cr }}$ and

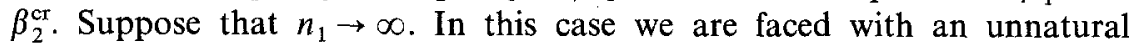
situation: $\beta_{2}^{\text {cr }} \rightarrow \infty$ while $\kappa_{2} \rightarrow \kappa_{1}$ and $\beta_{1}^{\text {cr }}$ is fixed.

The reason for this situation is the following fact: the Peierls constant $t$ (see Lemma 1) for the special ground state in this case tends to zero when 
$n_{1} \rightarrow \infty$. Correctness of the last fact can be easily shown directly from the construction of the special ground state. Indeed,

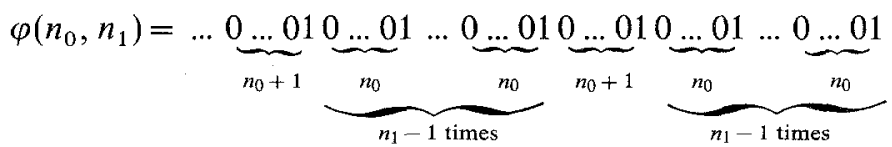

$$
\begin{aligned}
& \times \underbrace{0 \ldots 01}_{n_{0}+1} \underbrace{\underbrace{0.001}_{n_{0}} \ldots \underbrace{0 \ldots 01}_{n_{0}} \ldots}_{n_{1}-1 \text { times }}
\end{aligned}
$$

Let us consider the following perturbation of the $\varphi\left(n_{0}, n_{1}\right)$ :

$$
\begin{aligned}
\varphi^{\prime}\left(n_{0}, n_{1}\right)= & \ldots \underbrace{0 \ldots 0}_{n_{0}+1} \underbrace{0 \ldots 01}_{\underbrace{0 \ldots 01}_{n_{1}-1 \text { times }} \ldots \underbrace{0 \ldots 01}_{n_{0}} \overline{0.01}} \underbrace{0 \ldots 01 \ldots \underbrace{0 \ldots 01}_{n_{0}+1} \underbrace{0.01}_{n_{n_{1}-2 \text { times }}^{0 \ldots 0}}}_{n_{0}} \\
& \times \underbrace{0 \ldots 01}_{n_{0}+1} \underbrace{0 . \ldots}_{\underbrace{0 \ldots 01}_{n_{1}-1 \text { times }} \ldots \underbrace{0 \ldots 01 \ldots}_{n_{0}}}
\end{aligned}
$$

which is obtained from the configuration $\varphi\left(n_{0}, n_{1}\right)$ by interchanging two blocks (these blocks are noted above by an overbar).

Now note that

$$
\Delta H=H\left(\varphi^{\prime}\left(n_{0}, n_{1}\right)\right)-H\left(\varphi\left(n_{0}, n_{1}\right)\right)<\operatorname{const}\left(n_{0} n_{1}\right)^{-2-\alpha}
$$

and therefore $\Delta H \rightarrow 0$ when $n_{1} \rightarrow \infty$.

By modification of this construction we can obtain an example where there exists an unbounded perturbation with bounded loss of energy when $n_{1} \rightarrow \infty$.

Inequality (104) does not allow us to generalize Theorem 3 for the irrational values of the density.

But we think that Theorem 3 is valid for irrational values of the density, too. To establish this fact one needs to improve the proof of Theorem 3 (actually, to improve the definition of a precontour).

\section{ACKNOWLEDGMENT}

I would like to thank Yavuz Nutku for kind hospitality at Bilkent University, where this paper was written. 


\section{REFERENCES}

1. Ya. G. Sinai, Theory of Phase Transitions. Rigorous Results (Acad. Kiado, Budapest, 1982).

2. S. E. Burkov and Ya. G. Sinai, Uspekhi. Mat. Nauk 38:205-225 (1983).

3. R. L. Dobrushin, Teor. Veroyat. Primenenie 18(2):201-229 (1968).

4. R. L. Dobrushin, Funk. Anal. Pril. 2(4):44-57 (1968).

5. D. Ruelle, Commun. Math. Phys. 9:267-278 (1968).

6. F. Dyson, Commun. Math. Phys. 12(2):91-107 (1969).

7. F. Dyson, Commun. Math. Phys. 21:269-283 (1971).

8. J. Hubbard, Phys. Rev. B 17:494-505 (1978).

9. P. L. Pokrovsky and G. V. Uimin, J. Phys. C. Solid State Phys. 11:3535-3549 (1978).

10. P. Bak, Incommensurate, commensurate and chaotic phases, Preprint Nordita (1982).

11. A. A. Kerimov, Teor. Mat. Fiz. 58(3):473-480 (1984).

12. P. Bak and R. Bruinsma, Phys. Rev. Lett. 49:249-252 (1982).

13. S. E. Burkov and Ya. G. Sinai, Sov. Sci. Rev. C 5 (1985).

14. A. A. Kerimov, Sov. Sci. Rev. C 5 (1985).

15. J. Bricmont, K. Kuroda, and J. L. Lebovitz, Commun. Math. Phys. 101:501-538 (1985).

16. A. A. Kerimov, J. Stat. Phys. 52(1/2):69-98 (1988).

17. R. L. Dobrushin, Funk. Anal. Pril. 2:31-43 (1974). 